\title{
Pion physics in the Liège intranuclear cascade model
}

\author{
Th. Aoust ${ }^{1,2}$ and J. Cugnon ${ }^{2}$ \\ ${ }^{1}$ SCK-CEN, Boeretang 200, B-2400 Mol, Belgium \\ ${ }^{2}$ University of Liège, Physics Department, allée du 6 Août 17, bât. B5, B-4000 Liège 1, Belgium
}

(Received 7 June 2006; published 12 December 2006)

\begin{abstract}
The implementation of $\pi$ production in the Liège intranuclear cascade model (INCL4) for spallation reactions is revisited to alleviate the overestimate of the $\pi$ yield. Three modifications are proposed for this purpose: a better $\pi N$ cross section at high energy, the introduction of a $\pi$ average potential, and the modification of the average mass of the $\Delta$ resonance. The $\pi$ potential is determined from a global fit of a set of data bearing on $\pi$ production in proton-induced reactions, on $\pi$-nucleus and absorption cross sections, and on proton production in $\pi$-induced reactions. The resulting $\pi$ potential is poorly determined in the nuclear interior and agrees with the phenomenological optical-model potentials in the surface region. With these modifications, the predictions of the INCL4 model concerning $\pi$ production cross sections in proton-induced reactions are considerably improved. Predictions of the improved version for $\pi$-nucleus reaction and absorption cross sections and for proton, residue, and fission cross sections in $\pi$-induced reactions are also presented and shown to give reasonably good agreement. Neutron production and some aspects of fission in $\pi$-induced reactions are also investigated and reasonably well predicted. Effects on the modifications on observables, which are not directly linked with $\pi$ 's, such as the neutron yield and the residue mass and charge spectra in proton-induced reactions are also investigated and shown to improve the description of these observable quantities. Several results on $\pi$ production and the relative insentivity to the $\pi$ potential in the nuclear interior are shown to be consistent with the fact that most $\pi$ 's are not produced in early collisions. Importance of rescattering in $\pi$ absorption on nuclei is also pointed out. A comparison is made with the so-called $\Delta$-hole model. Residual discrepancies are identified and are interpreted as due to the lack of $\pi$ interaction with two nucleons at low energy, to the neglect of quantum motion effects, and to a possible underestimate of rescattering.
\end{abstract}

DOI: 10.1103/PhysRevC.74.064607

PACS number(s): 25.40.Sc, 25.40.Qa

\section{INTRODUCTION}

The interest in spallation reactions has recently been revived by the prospect of using accelerator-driven systems (ADS) as incinerators of nuclear waste [1], by projects of intense spallation sources [2], and by radioprotection issues regarding cosmic rays in space missions [3]. The intranuclear cascade+evaporation models, which are the standard theoretical tools for the description of spallation reactions (at least in the $\mathrm{GeV}$ range) are thus becoming more and more refined. In particular, it has been shown that the intranuclear cascade model developed at the University of Liège, denoted INCL4, coupled to the evaporation-fission model of Karl-Heinz Schmidt [4,5], can describe quite well, without fitting parameters, a huge body of experimental data for proton-induced reactions in the $100-\mathrm{MeV}$ to $2-\mathrm{GeV}$ range [6]. This includes total reaction cross sections, neutron multiplicities, proton and neutron double differential cross sections, residue mass spectra, isotopic distributions, and recoil energies.

The agreement is though not perfect: a few systematic discrepancies have been identified. For instance, this model has the tendancy to overpredict $\pi$ production by a factor that can raise to $1.3-1.8$ at $730 \mathrm{MeV}$. This discrepancy may not appear as too surprising, because pion emission is a complicated process. Emitted $\pi$ 's are not produced directly. They result from a succession of creations and absorptions inside the nucleus. Only those $\pi$ 's that are produced close to the surface have a good chance to be emitted. Therefore, the production mechanism by multiple collisions is presumably sensitive to the facets of the $\pi$ and $\Delta$ resonance dynamics. We want here to reconsider the $\pi$ production model that is implemented in the INCL4 model on three points: $\pi N$ cross section, introduction of $\pi$ average potentials, and modification of the $\Delta$ parameters due to in-medium effects. Concerning the second and third points, our original intention was to inject as much as possible information from phenomenology and/or theoretical guidelines. As we explain below, the information is rather poor on some important elements. Therefore we were forced to rely on a pragmatic approach. We introduce $\pi$ potentials and changes of some of the $\Delta$ parameters and determine them by a rough global fit of various $\pi$ production data in proton-induced reactions. We nevertheless constrained this fit quite a bit by also reproducing $\pi$-nucleus reactions, absorption cross sections, and proton spectra in $\pi$-induced reactions at the same time.

Our primary concern is to improve the physics carried by the INCL4 model. But we are also motivated by the importance of $\pi$ production in applications. Just to mention one aspect, namely the neutron yield of a thick spallation target bombarded by a proton beam, neglecting pion production in elementary collisions may underestimate the neutron yield, as we explain below.

The article is organized as follows. In Sec. II, we elaborate a bit on the relevance of $\pi$ production for spallation targets. In Sec. III, we briefly present the INCL4 model and the $\pi$ 

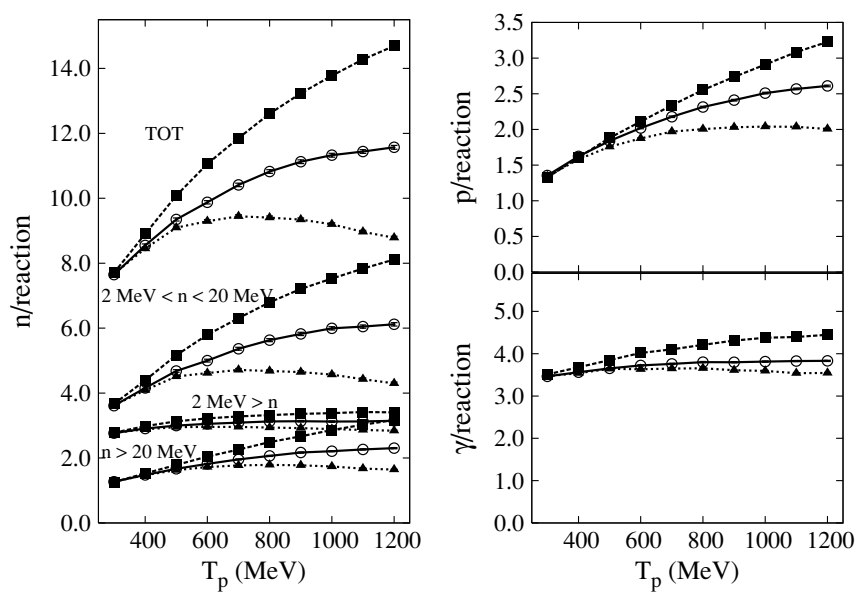

FIG. 1. Influence of $\pi$ production on neutron, proton, and $\gamma$ multiplicities (per reaction) in proton-induced reactions on $\mathrm{a} \mathrm{Pb}$ thin target, as functions of the incident proton kinetic energy $T_{p}$. The lines with the squares refer to the standard INCL4 calculations. The lines with the triangles are obtained by suppressing the inelastic $N N$ channel and the lines with circles correspond to keeping the same total $N N$ cross section but assuming purely elastic collisions. For neutrons, total multiplicities as well as multiplicities for three energy ranges are displayed.

production scenario that is implemented. We describe, in Sec. IV, the improvements of this scenario that we want to include. In Sec. V, we present our determination of the $\pi$ average potential. Section VI describes our results with the improved version of INCL4. They are compared with the results of the standard version and with experiment. Section VII contains a short discussion of our results. Finally, Sec. VIII presents our conclusion.
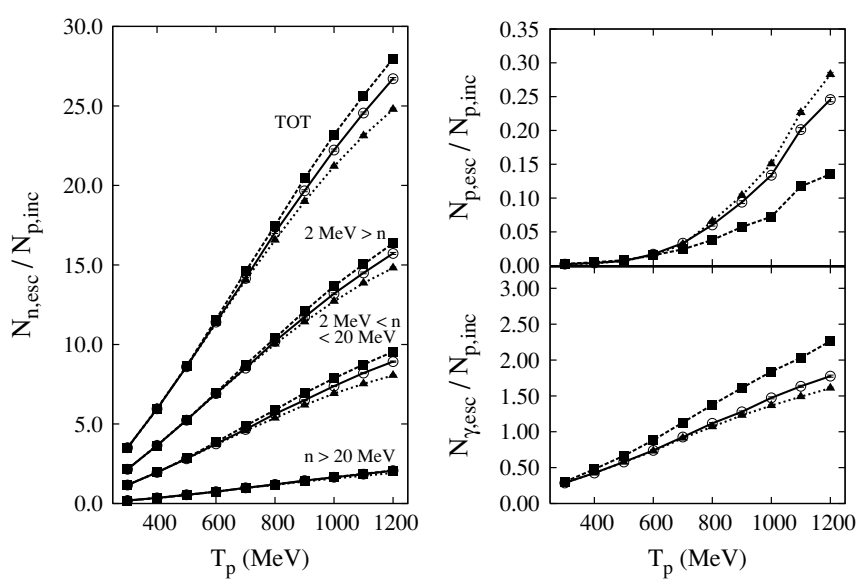

FIG. 2. Same as described in the legend to Fig. 1 for a thick cylindrical $\mathrm{Pb}$ target $($ diameter $=20 \mathrm{~cm}$, length $=60 \mathrm{~cm})$. Multiplicities of particles escaping from the target, per incident proton, are displayed. Same conventions as in Fig. 1.

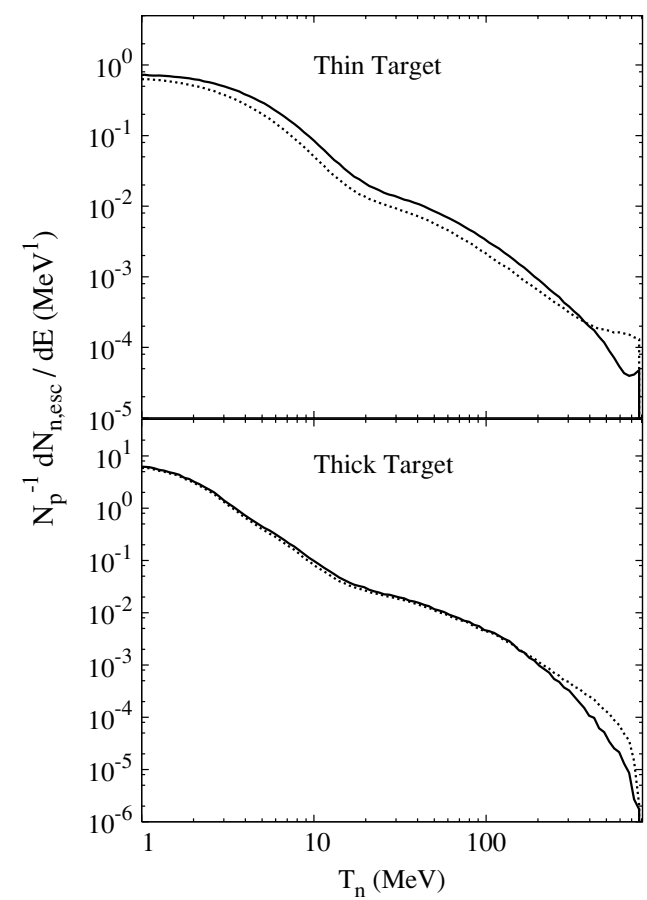

FIG. 3. Energy differential neutron multiplicity (per incident proton) induced by $800-\mathrm{MeV}$ protons on a thin (upper panel) or thick (lower panel) $\mathrm{Pb}$ target. The full lines refer to the standard INCL4 calculation, whereas the dotted lines correspond to suppressing the $N N$ inelastic channel.

\section{IMPORTANCE OF PIONS FOR SPALLATION TARGETS}

To assess the importance of $\pi$ production for spallation reactions, in Figs. 1-3 we compare some physical quantities calculated with or without $\pi$ production. We made a full calculation with INCL4 and compare it with a calculation with a vanishing inelastic nucleon-nucleon cross section (in the energy range under consideration, $\pi$ production exhausts the inelastic cross section). We also consider a calculation with the same assumption, but assuming the elastic cross section to be equal to the experimental total cross section. This option is perhaps more meaningful, as the "reactivity" of the nucleons is kept the same but is then purely elastic. Figure 1 refers to thin target calculations. One can see that suppressing $\pi$ production may have dramatic effects on the proton and especially the neutron yields. The $\gamma$ yield (including also hard $x$ rays) is less affected. Of course, the effect is increasing with incident energy. When inelasticity is suppressed while keeping the same total $N N$ cross section, the effect is roughly reduced by half. It is also interesting to note that the effect is the largest for evaporated neutrons. This is probably linked with the fact that (in INCL4) $\pi$ production proceeds via $\Delta$ production. Due to the large mass of the $\Delta$, this process is very efficient for transforming the available incident energy to excitation energy, as it is well known for a long time [7]. In Fig. 2, we make the same comparison for a thick target, a 60-cm-long cylinder, made of $\mathrm{Pb}$, with a $10-\mathrm{cm}$ radius. We concentrate on particles escaping from the target. The difference between the results with or without $\pi$ production is much reduced. This is due to 
secondary reactions. If $\pi$ production is suppressed, the incident particle transfers less energy in the first interaction, but this is compensated by the possibility of making several subsequent interactions. What matters finally for neutrons and protons is roughly the available energy. Suppressing $\pi$ production may still reduce the total neutron yield by $10 \%$ or more above $1 \mathrm{GeV}$. Neglecting inelasticity (keeping the same total $N N$ cross section) makes a $5 \%$ reduction in the same region. The effect on the proton yield is more subtle. Few protons are getting out anyway, because they are stopped inside the target. When the inelastic channel is simply suppressed, the proton yield is increased. This comes from the fact that protons have then less chance to interact, because the total cross section is reduced. When the inelasticity is cut while keeping the total cross section the same, the proton yield is reduced, in comparison to the previous case, as expected. The proton yield is nevertheless increased compared to the standard case. Even if the total cross section is the same, the inelasticity is nevertheless reduced, protons are thus more energetic on the average and have more chance to escape. The effect on the $\gamma$ yield amounts to $20 \%-30 \%$ around $1 \mathrm{GeV}$. The escaping $\gamma$ 's are coming from the deexcitation of the remnants and from $\pi^{0}$ decays. Because they are very hard, these $\gamma$ rays are important for shielding issues. The reduction when $\pi$ production is suppressed is mainly explained by the removal of the $\gamma$ 's from $\pi^{0}$ decay.

We also tried to assess the importance of the secondary reactions induced by $\pi$ 's in a thick target. We made a calculation allowing $\pi$ production, but without transporting $\pi$ 's inside the target. In other words, we consider that they cannot interact. Results are not shown, but they are close to the results obtained with cutting the inelastic channel while keeping the same total $N N$ cross section.

We want also to stress that $\pi$ production may have some drastic effect on residue production in thick target experiments. Just to mention an example: most of spallation targets are made of $\mathrm{Pb}$ or $\mathrm{Pb}-\mathrm{Bi}$ and highly radiotoxic $\mathrm{Po}$ isotopes can be made by $\left(p, \pi^{0}\right)$ or $\left(p, \pi^{-}\right)$reactions.

In Fig. 3, we show the effect of suppressing $\pi$ production on the neutron spectra. Both for thin and thick targets, the spectra are slightly hardened. For thin targets, the effect amounts mainly to an overall reduction of the neutron spectrum, in concordance with Fig. 1.

\section{PION DYNAMICS IN THE LIÈGE INTRANUCLEAR CASCADE MODEL}

We refer to Ref. [6] for a detailed description of INCL4. It is sufficient here to remind that it is a timelike simulation model, where all particles are followed in space-time. Particles are subject to collisions when they reach their minimum relative distance of approach, provided the latter is small enough compared to the square root of the cross section divided by $\pi$ and provided the final states are allowed by the Pauli principle. The model has a self-consistent criterion for stopping the cascade, which corresponds, on the average, to a randomization of the emission pattern. It should be mentioned that for this work a careful determination of the stopping time for $\pi$-induced cascades has been done, similarly to what is described in Ref. [6] for nucleon-induced cascades.

Let us give a little bit of detail on the implementation of $\pi$ production. Pions are supposed to be produced in two steps: $N N \rightarrow N \Delta$ and $\Delta \rightarrow N \pi$. Pion absorption is assumed to proceed via the reverse reactions. This looks reasonable, because $\Delta$ production dominates the inelastic $N N$ channel below $2 \mathrm{GeV}$, at least in the $p p(T=1)$ channel, and because the $\pi N$ interaction is dominated by the $\Delta$ resonance (note that, in INCL4, the $p n$ inelastic channel is assumed to proceed through the same reactions, which is critizable, because the $p n$ inelastic cross section contains a nonnegligible $T=0$ component). The $N N \rightarrow N \Delta$ cross section is taken as the total reaction cross section. The $N \Delta \rightarrow N N$ cross section is calculated from the previous one by detailed balance, empirically corrected for the lifetime of the $\Delta$. In $N N \rightarrow N \Delta$, the $\Delta$ is given a mass at random in a Breit-Wigner distribution, corrected by phase-space factor (and in agreement with conservation of energy) and a lifetime taken randomly in an exponential distribution, with an average of $\tau_{\Delta}=\hbar / \Gamma$ and $\Gamma=120 \mathrm{MeV}$. The $\pi^{+} p \rightarrow \Delta^{++}$ cross section is simply taken as the total experimental cross section:

$$
\sigma=\frac{326.5 q^{3}}{\left[1+4\left(\frac{\sqrt{s}-1215}{110}\right)^{2}\right]\left(q^{3}+180^{3}\right)}
$$

where $q$ is the $\pi$ momentum in the center of mass. This equation, where $\sigma$ is in $\mathrm{mb}, \sqrt{s}$ in $\mathrm{MeV}$ and $q$ in $\mathrm{MeV} / c$, fits the data rather nicely in the resonance region and more precisely for $\sqrt{s} \lesssim 1.5 \mathrm{GeV}$. In INCL4, this cross section is also used at higher energy, where, of course, it departs from experimental data.

The elastic $N \Delta$ cross section is taken equal to the $N N$ elastic cross section at the same center-of-mass energy. Isospin conjugation is used as far as possible for determining the cross sections that are not measured experimentally.

In INCL4, $\Delta$ particles are moving in the same potential as the nucleons (the same for protons and neutrons). They can be reflected or transmitted at the nuclear surface, according to the same rules as for nucleons. Pions do not feel any potential.

\section{IMPROVEMENTS OF PION DYNAMICS}

\section{A. Introduction}

In this article, we try to improve the implementation of $\pi$ dynamics on three aspects. We first complete the proper parametrization of the $\pi N$ cross section above the $(3,3)$ resonance. Second, we introduce an average potential well for $\pi$. Third, we also introduce some in-medium modifications of some cross sections and of the parameters of the $\Delta$ resonance. But, in view of the poor documentation of this last point, we limit ourselves to just a few exploratory sensitivity studies. Compared to the standard INCL4 version of Ref. [6], we also introduced isospin-dependent and energy-dependent nuclear 
potentials for baryons, in exactly the same way as in Ref. [8]. The effects of this modification on $\pi$ observables are, in general, rather minute. Below, we comment on theses effects only when they are not negligible.

\section{B. The $\pi N$ cross section}

We introduce a parametrization of the $\pi^{+}-p$ and $\pi^{-}-p$ cross sections that fit the experimental data up to $5 \mathrm{GeV}$, as shown in the Appendix. Other cross sections are obtained by isospin symmetry. We continue to consider that the $\pi$ and the nucleon form an object with a mass equal to their center-ofmass energy. The average lifetime of this object is taken equal to the $\Delta$ lifetime in the $\Delta$ resonance region and to half of this value outside this region (to account for the nonresonant scattering). In this way, elastic scattering appears in a two-step process, which is, for us, technically easier. We thus disregard $\pi N$ inelastic scattering. This process is negligible for $\pi$ 's with kinetic energy less than $500 \mathrm{MeV}$, which are basically outside our energy range of interest here. Below the $\Delta$ resonance region, a cross section of $5 \mathrm{mb}$ is imposed for all $\pi$ 's. This helps to mock up the direct absorption on a correlated pair of nucleons, which may have some secondary importance at low energy, as suggested by the $\pi^{+}+d \rightarrow p p$ process that exhibits a few-mb cross section in this particular range of energy. We may anticipate by saying that this refinement has very little importance on our results below.

\section{Pion average potential}

We want to introduce the average potential energy felt by a $\pi$ sitting inside the nucleus. The first idea is to turn, as for nucleons, to the phenomenology of the $\pi$ optical-model potential and to identify the $\pi$ average potential energy to the real part of the $\pi$-nucleus optical-model potential. This raises a number of difficulties. First, many optical-model analyses are based on nonlocal potentials, such as the popular Kisslinger form [9], drawing their nonlocality from their connection with the elementary $\pi N$ amplitude, made of $s$ and $p$ components. Nonlocal potentials are not appropriate for a classical treatment as implied by our simulations. Fortunately, Kisslinger-type potentials can be cast into a local form, owing to a KrellEricson transformation [10]. Second, usual optical-model potentials, even local ones, exhibit complicated geometry and fits are not always done with a standard geometry, as for the nucleon optical-model potential. Furthermore, these potentials often exhibit a continuous ambiguity in the determination of the parameters [11], especially when the coupling to collective low-lying states is strong [12]. For this reason, some analyses have been performed using so-called "bias-free" geometry, i.e., Bessel-Fourier series [13]. Grossly speaking, these analyses show that the real part can be put into a sum of volume and surface components, corresponding to radial distances smaller and larger than the half-density radius. Results of fits show scattered values of the real part of the optical-model potential. Nevertheless, some systematic properties emerge, as illustrated in Fig. 4: (a) the real potential is negative in the

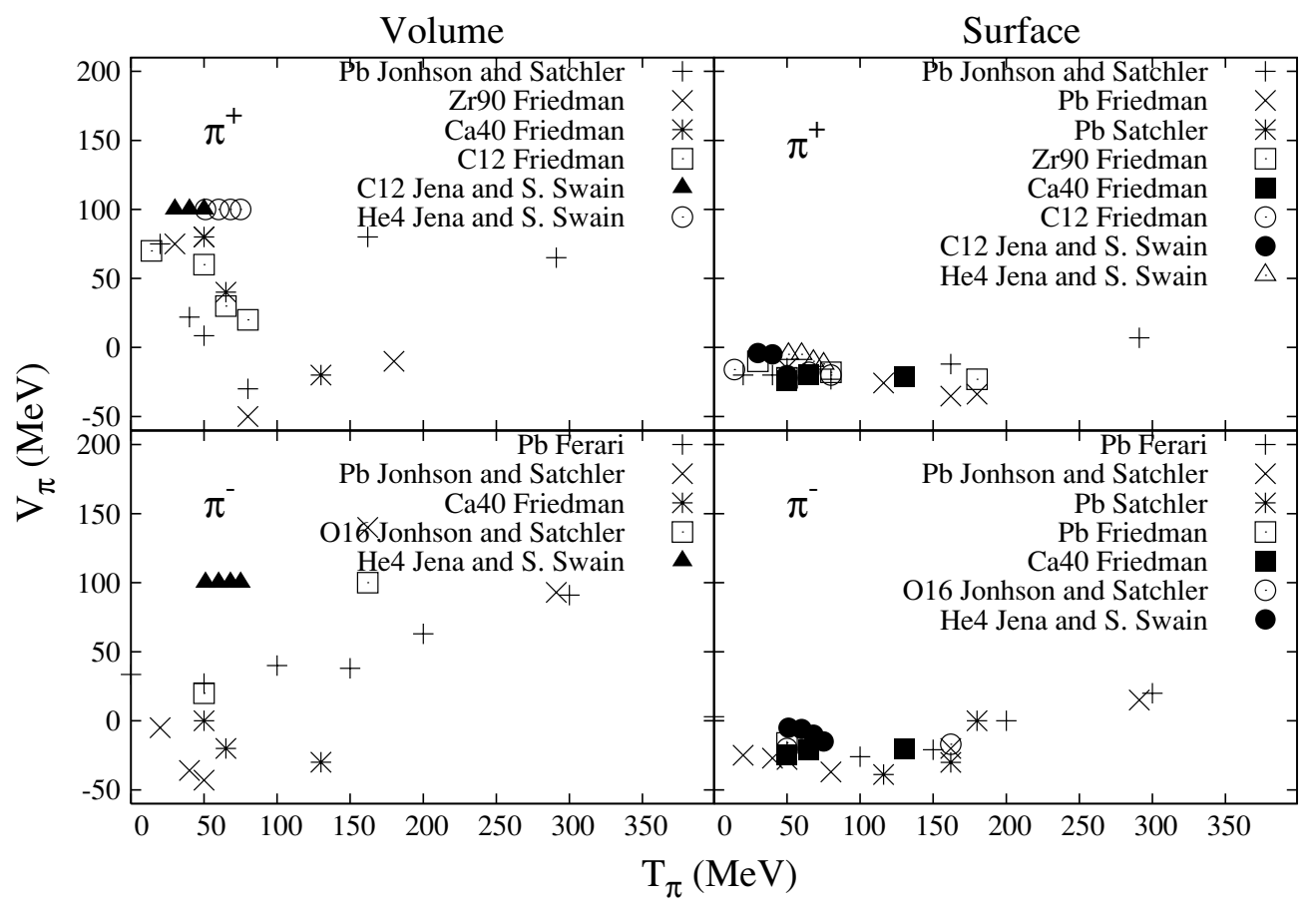

FIG. 4. Phenomenology of the real part of the $\pi$ optical-model potential based on local potentials, composed of a volume (left panels) plus a surface (right panels) components (the decomposition is either taken from the indicated references or made by us as a smooth separation around the half-density radius). The quantity $T_{\pi}$ is the $\pi$ kinetic energy. The upper (lower) panels refer to positive (negative) $\pi$ 's. Experimental data are taken from Refs. $[11,13-15]$. The convention corresponds to negative (positive) values for attractive (repulsive) potentials. 
surface region; (b) in this region, it is fairly independent of the incident energy below $100 \mathrm{MeV}$, above which its absolute value tends to decrease; (c) in the nuclear interior, the real potential is positive for low-energy positive $\pi$ 's and is roughly vanishing for low-energy negative $\pi$ 's; (d) in the same region, as the $\pi$ energy increases, the $\pi^{+}$potential deacreases fairly rapidly and vanishes somewhere between 80 and $120 \mathrm{MeV}$; and (e) in the resonance region, the real potential in the interior is badly determined. The data in Fig. 4 also suggest a slight dependence on the charge asymmetry of the target and on the charge of the $\pi$ for the surface potential. The imaginary part of the optical-model potential (not shown) is negative, as expected. It is rather important, and does assume a smooth geometry: it is roughly proportional to the density. Furthermore, it shows a resonating energy dependence, being maximum around $200 \mathrm{MeV}$, corresponding to the location of the $(3,3)$ resonance [13]. This brings to the third difficulty. The real part of the optical-model potential (in the interior at least) is badly determined by optical-model fits, because, due to the large absorption, the elastic part of the wave function does not probe really the interior. In addition, the strong resonating absorption (of the elastic component) induces, through the dispersion relation that real and imaginary parts have to fulfill [16], a strong energy dependence of the real part, which is visible in Fig. 4, in spite of the poor determination of this potential in the interior of the nucleus. This sheds some doubt on the physical meaning of the real part, at least on its interpretation in terms of the potential energy of a $\pi$. Indeed, the optical model just describes the flux in the elastic channel and certainly does not refer to the potential energy of a $\pi$ created inside the nuclear volume, which is precisely what we are after. This potential energy is not tested by elastic scattering. However, it can in principle be tested in $\pi$-producing reactions.

In view of this discussion, we decided to disregard the phenomenology of real part of the optical-model potential and to use $\pi$ potentials with the same amplitude in the surface and in the volume. Actually, we just consider the following simple form

$$
\begin{aligned}
& V\left(r, \tau_{3}\right)=V_{t}\left(\tau_{3}\right)=V_{N}\left(\tau_{3}\right)+\bar{V}_{C}, \quad \text { for } \quad r<R_{c}, \\
& V\left(r, \tau_{3}\right)=V_{C}(r)=\frac{Z_{T} \tau_{3} e^{2}}{r}, \quad \text { for } \quad r>R_{c} .
\end{aligned}
$$

In this equation, $\tau_{3}$ is the third component of the $\pi$ isospin and $Z_{T}$ is the (actual) target charge number. For $r>R_{c}$, the potential reduces to the Coulomb potential. For $r$ smaller than $R_{c}$, the potential has a constant value, which is the sum of a nuclear component $V_{N}$ and of an average Coulomb component $\bar{V}_{C}$. The first component takes a form similar to the Lane model for nucleon-nucleus interaction [17]:

$$
V_{N}\left(\tau_{3}\right)=V_{N}^{0}+V_{N}^{1} \tau_{3} \xi,
$$

where $\xi=(N-Z) / A$ is the asymmetry parameter of the target. Finally, $\bar{V}_{C}$ is the average Coulomb interaction that we ascribe to

$$
\bar{V}_{C}=\tau_{3} \frac{1.25 Z_{T} e^{2}}{R_{0}},
$$

where $R_{0}$ is the half-density radius. The quantity $R_{c}$ is taken as $R_{0}+2 \mathrm{fm}$, somehow as indicated by the phenomenology.
Facing the uncertainty of experimental knowledge, we finally decided to treat $V_{N}^{0}$ and $V_{N}^{1}$ as phenomenological parameters and tried to determine them by a rough fit of the experimental data, not only for total $\pi N$ reaction cross sections (as for the optical-model potential) but also for a whole set of observables implying $\pi$ 's as incident or produced particles (see below for details). We, however, require the depth of $V_{N}$ to be not too far from the phenomenological values for the potential at the surface, which seems more solidly founded.

In our approach, we do not need any imaginary part, as the inelastic processes, including true absorption, are explicitly described (through incoherent $\pi N$ collisions). Finally, $\pi$ 's are transmitted through the external Coulomb barrier in the same way as the protons (see Ref. [6]).

\section{Modification of the $\Delta$ parameters}

The in-medium modification of the $\pi$ and $\Delta$ properties are often regarded as important features [18]. Here, we look at the influence of the parameters of the $\Delta$ resonance, as implemented in our model. These parameters include the average mass, the width of the mass distribution and the average lifetime. Possible modifications of these parameters, due to in-medium effects, are difficult to relate to experimental and theoretical knowledge. For instance, the lifetime of the $\Delta$ resonance is expected to be enhanced by the Pauli principle and to be reduced by $\Delta N \rightarrow N N$ process. But because we explicitly account for these two effects, it seems reasonable to keep the intrinsic lifetime unaffected. However, if we consider the stochastic choice of the $\Delta$ mass as simulating the $\Delta$-mass distribution, we may try to account for medium effects. It is sometimes considered that the shift of the peak (and the increase of the width of this peak of the total $\pi N$ cross section with the mass of the target), compared to the free $\pi N$ cross section, is a manifestation of the modification of the $\Delta$ properties inside matter [19]. However, one has to keep in mind that the differences can be due also to binding, off-shell, and multiple-scattering effects. It is perhaps better to look at the theoretical investigations of the $\Delta$ self-energy inside matter. Reference [20] contains probably the most reliable investigation of the problem and points to lowering of the (average) mass by $25 \mathrm{MeV}$ inside nuclear matter at normal density. Guided by this result, we investigate in this article the influence on our results of a possible lowering of the $\Delta$ mass by $50 \mathrm{MeV}$ at the most. We did not try to change the width of the $\Delta$-mass distribution. We will come back to this point.

\section{DETERMINATION OF THE PION POTENTIAL}

We determined the "optimal" $\pi$ potential by searching for the values of $V_{N}^{0}$ and $V_{N}^{1}$ that yield the best description of many measured quantities, using the INCL model. We based our study on the data quoted in Table I. Figure 5 gives an idea of the acceptable values of the above parameters, for different targets. A common choice can be identified as

$$
\begin{aligned}
& V_{N}^{0}=-30.6 \mathrm{MeV}, \\
& V_{N}^{1}=-71.0 \mathrm{MeV} .
\end{aligned}
$$


TABLE I. List of the experimental data used in the determination of the $\pi$ potential.

\begin{tabular}{lcc}
\hline \hline Reactions & Observables & References \\
\hline$p(500,730 \mathrm{MeV})+{ }^{208} \mathrm{~Pb}$ & $\sigma_{\text {prod }}\left(\pi^{+}\right), \sigma_{\text {prod }}\left(\pi^{-}\right)$ & {$[21,22]$} \\
$p(300,400,600,730 \mathrm{MeV})+{ }^{12} \mathrm{C}$ & $\sigma_{\text {prod }}\left(\pi^{+}\right), \sigma_{\text {prod }}\left(\pi^{-}\right)$ & $p$ spectra $T_{p}>40 \mathrm{MeV}$ \\
$\pi^{+}(100,160,220 \mathrm{MeV})+{ }^{\text {nat }} \mathrm{Ni}$ & $p$ spectra $T_{p}>40 \mathrm{MeV}$ & {$[21-23]$} \\
$\pi^{+}(100,160,220 \mathrm{MeV})+{ }^{181} \mathrm{Ta}$ & $p$ spectra $T_{p}>40 \mathrm{MeV}$ & {$[24]$} \\
$\pi^{-}(100,160,220 \mathrm{MeV})+{ }^{n a t} \mathrm{Ni}$ & $p$ spectra $T_{p}>40 \mathrm{MeV}$ & $\sigma_{\mathrm{abs}}, \sigma_{R}$ \\
$\pi^{-}(100,160,220 \mathrm{MeV})+{ }^{181} \mathrm{Ta}$ & $\sigma_{\mathrm{abs}}, \sigma_{R}$ \\
$\pi^{+}(85,125,165,205,245,315 \mathrm{MeV})+{ }^{12} \mathrm{C}$ & $\sigma_{\mathrm{abs}}, \sigma_{R}$ & {$[24]$} \\
$\pi^{+}(85,125,165,205,245,315 \mathrm{MeV})+{ }^{56} \mathrm{Fe}$ & $\sigma_{\mathrm{abs}}, \sigma_{R}$ \\
$\pi^{+}(85,125,165,205,245,315 \mathrm{MeV})+{ }^{209} \mathrm{Bi}$ & $\sigma_{\mathrm{abs}}, \sigma_{R}$ \\
$\pi^{-}(69.5,87.5,125,150,165,205,230,245,280,315 \mathrm{MeV})+{ }^{12} \mathrm{C}$ & $\sigma_{\mathrm{abs}}, \sigma_{R}$ \\
$\pi^{-}(125,165 \mathrm{MeV})+{ }^{56} \mathrm{Fe}$ & {$[24]$} \\
$\pi^{-}(125,165 \mathrm{MeV})+{ }^{209} \mathrm{Bi}$ & {$[25]$} \\
\hline \hline
\end{tabular}

This corresponds to a total nuclear potential of $-30.6 \mathrm{MeV}$ for ${ }^{12} \mathrm{C}$, irrespective of the charge of the $\pi$, and of approximately $-22,-30$, and $-38 \mathrm{MeV}$ for $\pi^{+}, \pi^{0}$, and $\pi^{-}$, respectively, in ${ }^{208} \mathrm{~Pb}$. It is rewarding to see that our procedure yields values that are roughly consistent with the phenomenological values of the $\pi$ potential at the surface (see Fig. 4). This seems to attach a physical meaning of the real part of the $\pi N$ optical-model potential as a potential energy in this region. However, the optimal values quoted above may not be equally satisfactory for all subsets of data. In addition, the description of some data is rather sensitive to changes of the value of the potentials, whereas others are not. We summarize the situation. Production of $\pi^{+}$'s $\left(\pi^{-}\right.$'s) in proton-induced reactions is rather independent of the value of negative (positive) $\pi$ potential. The description of $\pi^{+}$production is slightly better for a $\pi^{+}$ potential of $\sim-30 \mathrm{MeV}$ and is always better for light targets than for heavy ones. The description of $\pi^{-}$production is better for a $\pi^{-}$potential of $\sim-50 \mathrm{MeV}$ and is largely satisfactory

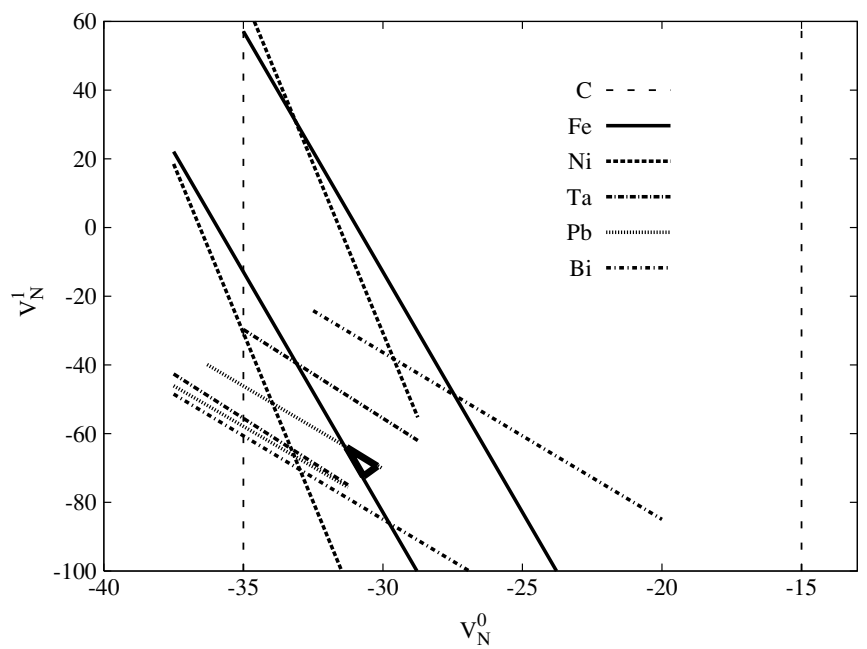

FIG. 5. For each nucleus, the area of possible $\left(V_{N}^{0}, V_{N}^{1}\right)$ values [see Eq. (3)] is delimited by similar line segments. They correspond roughly to to an increase of the $\chi$ squared by one unit. There is no dependence on $V_{N}^{1}$ for the (symmetric) ${ }^{12} \mathrm{C}$ target. The triangle with heavy lines is the overlap of all areas of acceptable values. for all targets. This is illustrated in Fig. 6. Positive $\pi$ reaction cross sections offer a good sensitivity to the $\pi^{+}$potential and point to a value of $\sim-20 \mathrm{MeV}$. Results are rather good for light targets and a little bit less satisfactory for heavy targets. Data on negative $\pi$ reaction cross sections are rather poor but
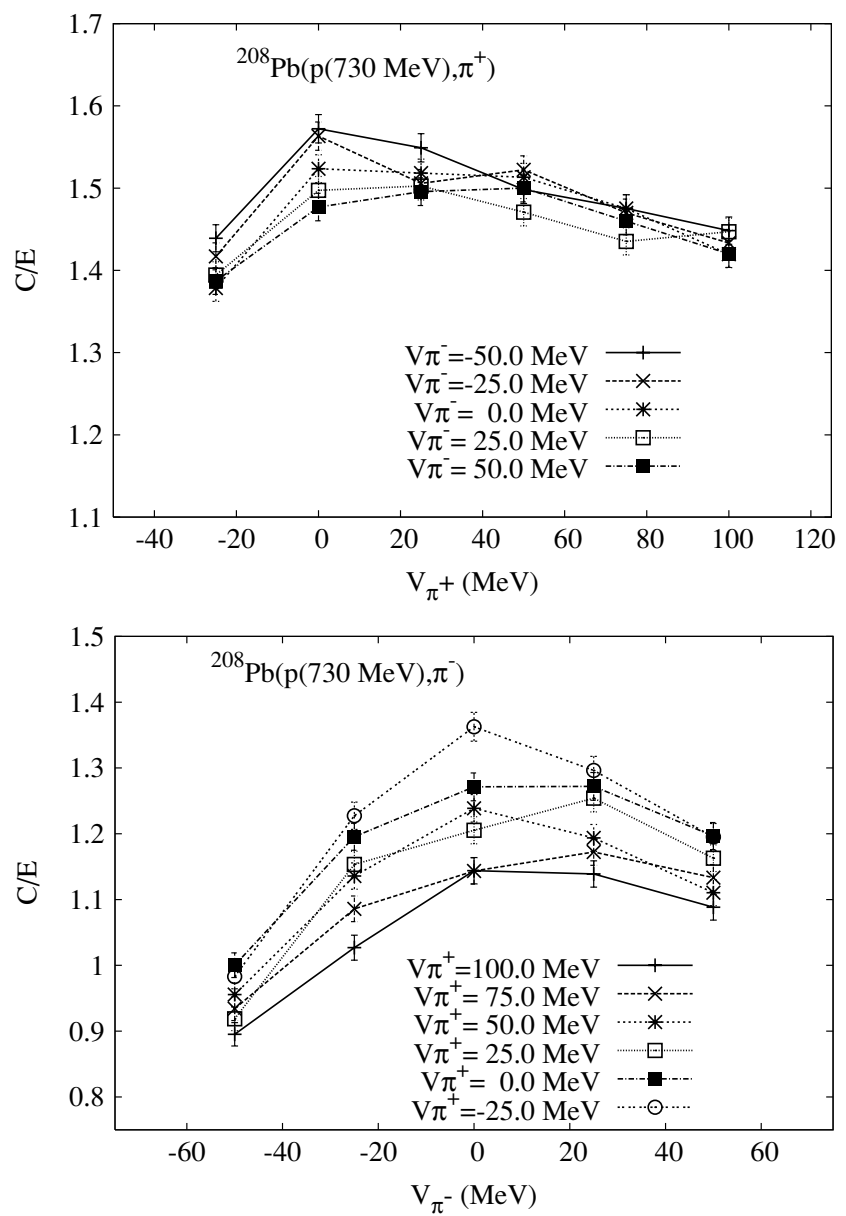

FIG. 6. Ratio of the calculated to the experimental $\pi^{+}$(up) and $\pi^{-}$(down) production cross section for reactions induced by $730-\mathrm{MeV}$ protons on $\mathrm{Pb}$, in function of the $\pi$ potentials implemented in the INCL model. Based on the experimental data from Ref. [22] 


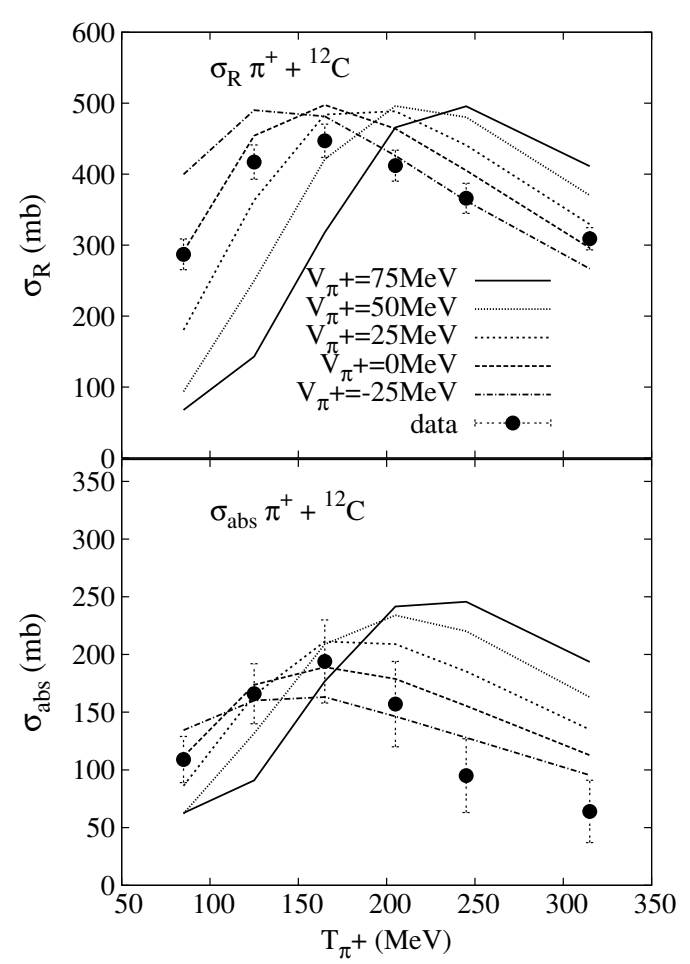

FIG. 7. Reaction (up) and absorption (down) cross section of positive pions on ${ }^{12} \mathrm{C}$ as a function of the $\pi$ kinetic energy $T_{\pi^{+}}$ for various values of $V_{\pi^{+}}$. The quantity $V_{\pi^{-}}$is kept equal to 0 . Experimental data are from Ref. [25].

yield to a similar conclusion, with a $\pi^{-}$potential close to the optimal value. True absorption cross sections are also rather sensitive to the $\pi$ potential. For $\pi^{+}$'s, the analysis roughly favors the value quoted above for the $\pi^{+}$potential in light targets, as shown in Fig. 7. In heavy targets, the same value also gives the most satisfactory description, although there remain significant deviations from experiment (see below). For $\pi^{-}$'s, the analysis yields a similar conclusion, pointing to a $\pi^{-}$potential of around $-50 \mathrm{MeV}$ in light and medium targets. Heavy target data do not allow any firm conclusion to be drawn. Finally, the production of protons in $\pi^{+}\left(\pi^{-}\right)$-induced collisions is manifestly insensitive to the value of the $\pi^{-}\left(\pi^{+}\right)$potential. In $\pi^{+}$-induced reactions, a $\pi^{+}$potential of $\sim-25 \mathrm{MeV}$ is the best choice and generates good results except for heavy targets. In $\pi^{-}$-induced reactions, a $\pi^{-}$potential of $\sim-50,-60 \mathrm{MeV}$ is better at high incident energy, but the description obtained this way is still unsatisfactory. At low incident energy, these values are still the best, but, surprisingly, a positive potential (of $20,30 \mathrm{MeV}$ ) works as well.

\section{ANALYSIS OF THE RESULTS}

We first discuss quantities that are directly influenced by the $\pi$ dynamics, namely $\pi$ production in nucleon-induced reactions and cross sections in $\pi$-induced reactions, including nucleon production cross sections. Afterwards, we also say a few words describing features of spallation reactions that are indirectly influenced by $\pi$ production scenario, such as

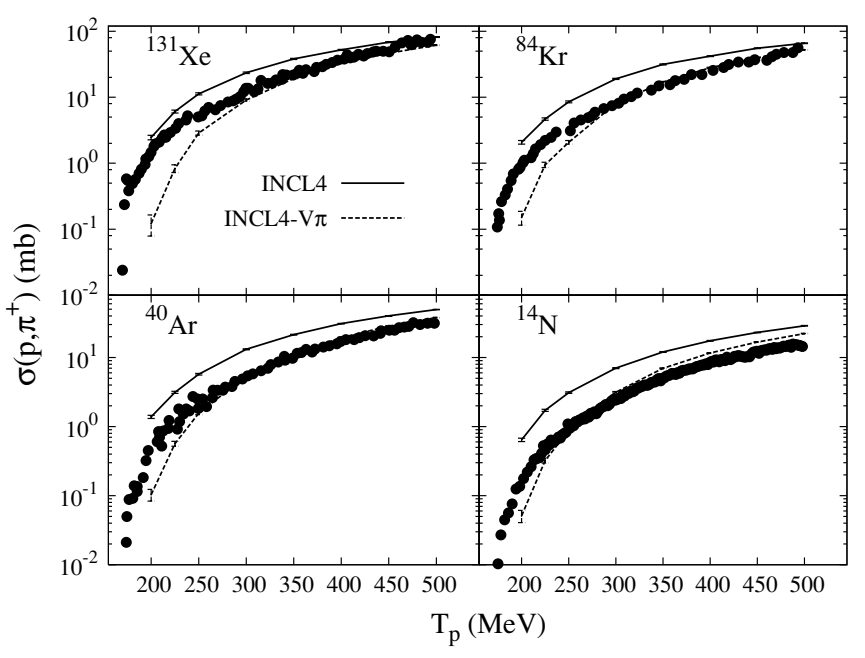

FIG. 8. Positive pion production cross-section in proton-induced reactions on various targets, as function of the incident proton kinetic energy $T_{p}$. The full lines are the standard INCL4 predictions. The dotted curves are obtained after introduction of the modified $\pi-N$ cross-section and of the pion potential (see text for detail). Experimental data are from Ref. [28]. Typical (statistical) uncertainties of the calculations are indicated by the error bars on the theoretical curves.

residue cross sections. Most of the displayed results have been obtained with the modified $\pi N$ cross section and the $\pi$ potential described in Secs. IV-V. We comment on the influence of the modification of the $\Delta$ mass only when it brings significant effects.

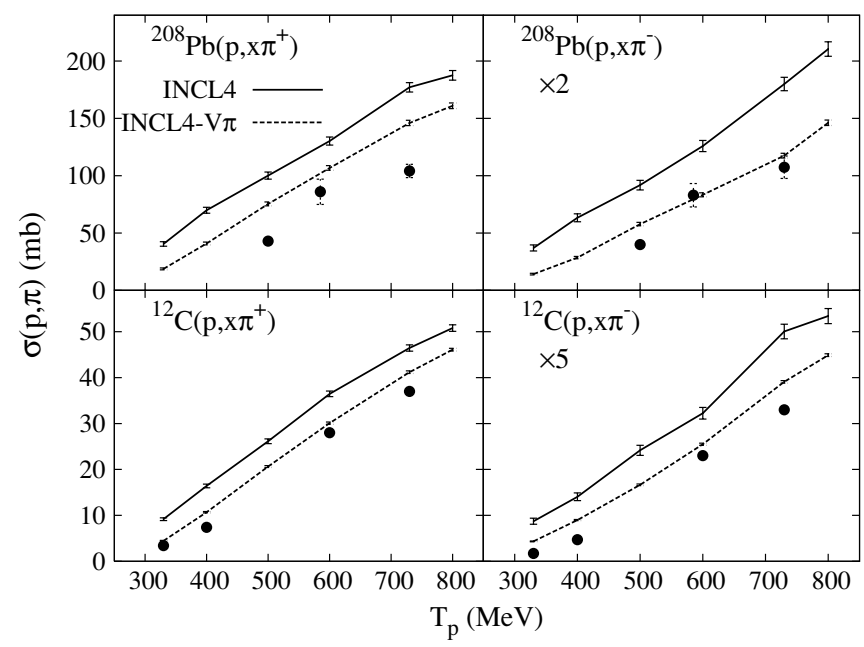

FIG. 9. Production cross-section of $\pi^{+}$'s (left) and $\pi^{-}$'s (right) in proton-induced reactions on ${ }^{208} \mathrm{~Pb}$ (up) and ${ }^{12} \mathrm{C}$ (down), as function of the incident proton kinetic energy $T_{p}$. Experimental data are from Refs. [21,22]. Full lines are the standard INCL4 predictions. The dotted curves are obtained after introduction of the modified $\pi-N$ cross-section and of the pion potential (see text for detail). Typical (statistical) uncertainties of the calculations are indicated by the error bars on the theoretical curves. 


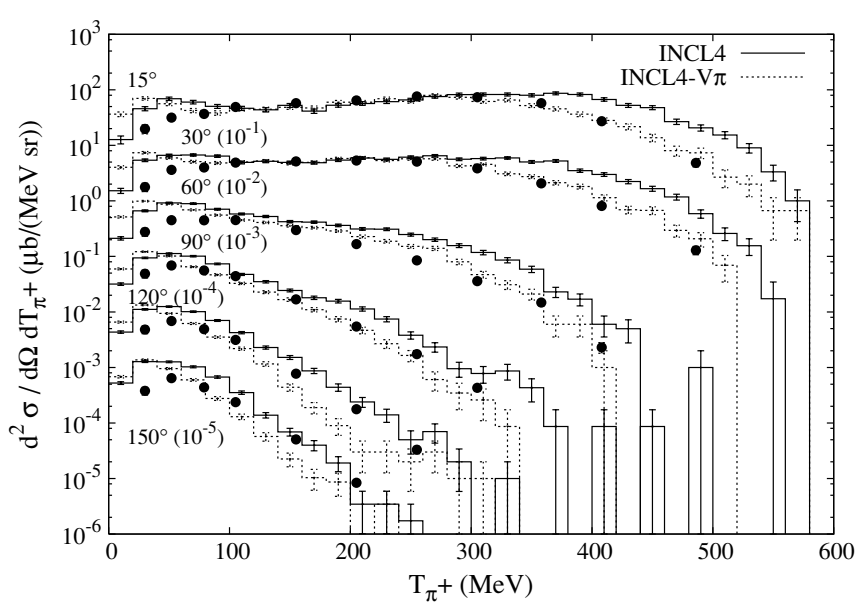

FIG. 10. Double differential production cross section of positive $\pi$ 's in proton-induced reactions on ${ }^{208} \mathrm{~Pb}$ at a kinetic energy of $730 \mathrm{MeV}$. Experimental data are from Ref. [22]. Full histograms correspond to standard INCL4 predictions. Dotted histograms are obtained after introduction of the modified $\pi N$ cross section and of the $\pi$ potential (see text for detail). To ease the reading of the figure, the successive spectra have been multiplied by decreasing powers of 10, except for the smallest angle, in which case absolute values are given. The values of the detection angles are displayed close to the corresponding curves. Typical statistical uncertainties on the theoretical calculations are indicated by the error bars on the histograms.

\section{A. Pion production in proton-induced reactions}

Total $\pi$ production cross sections on various targets have been calculated and compared with standard INCL4 calculations and with experimental data. In Fig. 8, we compare with the measurements of Ref. [28] on $\pi^{+}$production by protons of $150-500 \mathrm{MeV}$. It is rather clear that the predictions of INCL4 are considerably improved by the modifications brought into the model. There remains an underestimate of the cross sections, when the latter are smaller than $\sim 5 \mathrm{mb}$ and there is a slight overshooting at large incident energy for the ${ }^{14} \mathrm{~N}$ target.

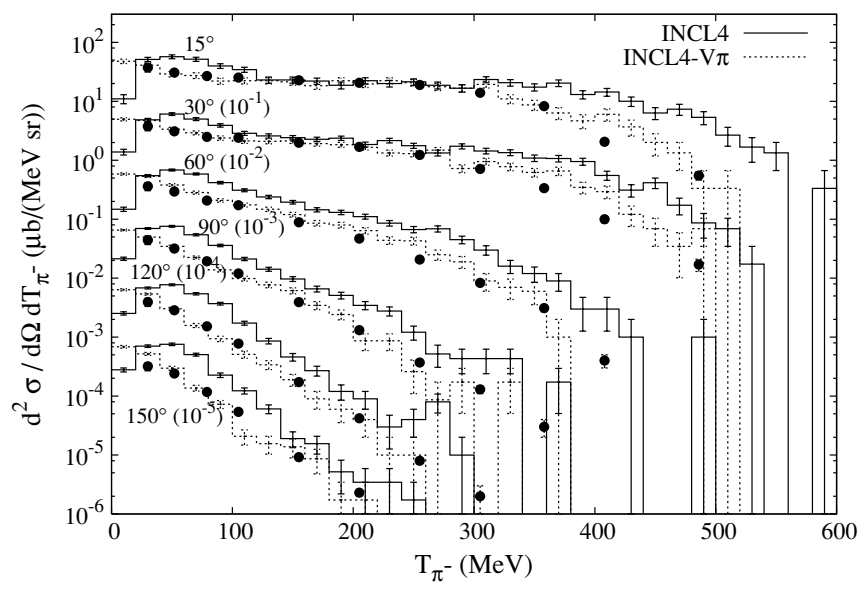

FIG. 11. Same as described in the legend to Fig. 10 for production of negative $\pi$ 's.

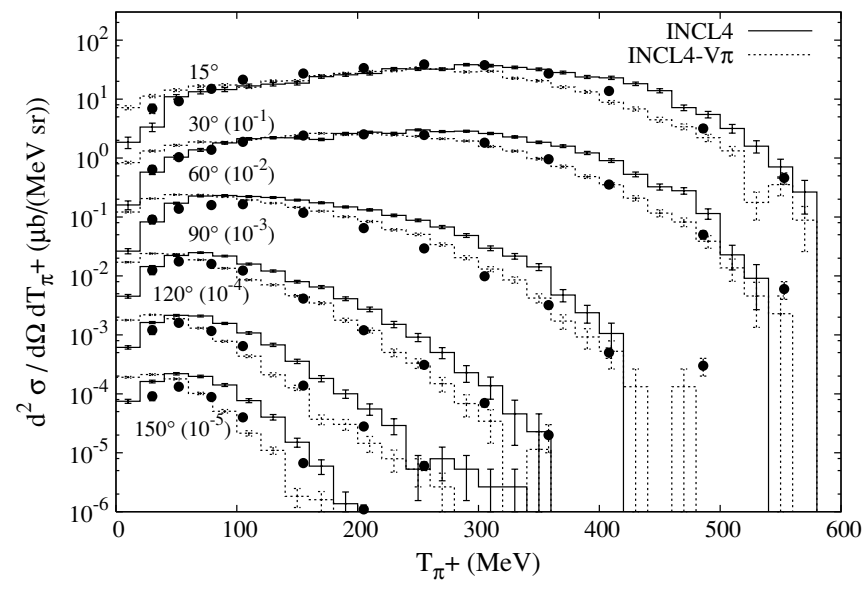

FIG. 12. Same as described in the legend to Fig. 10 for a ${ }^{12} \mathrm{C}$ target.

Figure 9 gives some account of the situation for higher incident energy. Here also, the results are sizably improved by the modifications described above and the agreement with experiment is quite good, except for $\pi^{+}$production on $\mathrm{Pb}$. Figures 10-13 display our results for $\pi$ double differential cross sections on ${ }^{208} \mathrm{~Pb}$ and on ${ }^{12} \mathrm{C}$ at $730 \mathrm{MeV}$. Let us comment each of them successively. In the $p+{ }^{208} \mathrm{~Pb} \rightarrow \pi^{+}+X$ case, the agreement with experiment is definitely improved when the modifications are incorporated. The emission of high-energy $\pi$ 's is sizably reduced, due to the introduction of the potential. The predictions are not really improved for low-energy $\pi$ 's. For $\pi^{-}$production on the same target, the agreement reached with the improved version is spectacular. One should notice, however, that the first bin of the histograms is noticeably enhanced with the modifed version. Unfortunately, this part of our results cannot be compared directly with experiment. Results for $\pi^{+}$and $\pi^{-}$production on ${ }^{12} \mathrm{C}$ are displayed in Figs. 12-13. The results with the modified version gives really accurate results.

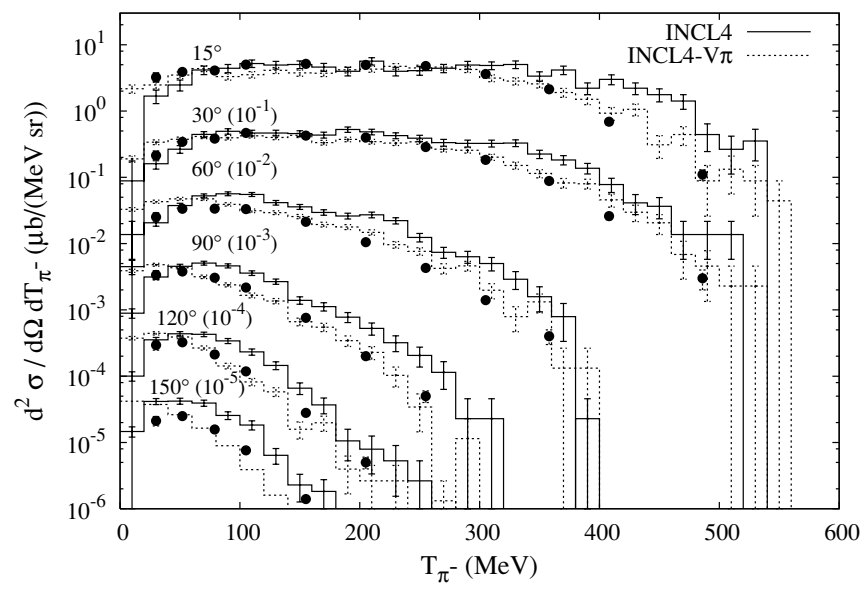

FIG. 13. Same as described in the legend to Fig. 12 for production of negative $\pi \mathrm{s}$. 


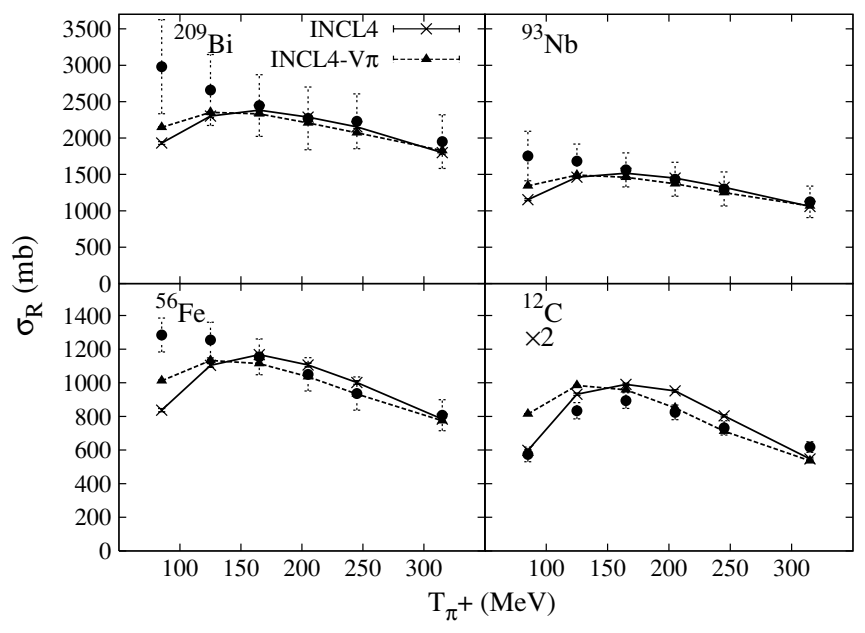

FIG. 14. Positive $\pi$ reaction cross section with four different targets. Comparison between the standard INCL4 predictions (full lines), the predictions after modification of the $\pi N$ cross section and introduction of the $\pi$ potential (see text for detail, dashed lines) and the experimental data (symbols) of Ref. [25]. Note that all cross sections for ${ }^{12} \mathrm{C}$ have been multiplied by 2 .

\section{B. Pion-nucleus reactions: Reaction and absorption cross sections}

We also tested our modifications of the model on the description of $\pi$-nucleus reactions. In Fig. 14, we focus on the total reaction cross sections. The agreement obtained with the standard version of INCL4 is already satisfactory and is only slightly improved by our modifications. This may rather be understandable, because what matters really is the first collision undergone by the incoming $\pi$ and because the associated cross section is not modified in the energy range under consideration. The fact that there is a maximum around $180 \mathrm{MeV}$ in the $\mathrm{C}$ cross sections means that the interaction is dominated by the $(3,3)$ resonance and the disappearance of this peak in heavier targets means that rescattering is more and more important as the target mass increases.

The general agreement that is reached indicates that we have picked up the main features of the $\pi$-nucleus interaction mechanism, but the lack of cross section at less than $100 \mathrm{MeV}$ for the heavy target (for $\pi^{-}$'s) may result from the neglect of direct interaction with two nucleons at low energy.

We turn to the (true) $\pi$ absorption cross section in Figs. 15-16. This is an important point, as the overestimate of $\pi$ production has often been attributed to the underestimate of $\pi$ absorption in the INCL4 scenario. In fact Fig. 15 reveals that $\pi^{+}$absorption is overestimated for incoming $\pi$ 's above $100 \mathrm{MeV}$ but may be substantially underestimated at low energy. The trend is even more accentuated for negative $\pi$ 's, as illustrated by Fig. 16. When the modifications are introduced (here essentially the $\pi$ potential), discrepancies are largely reduced. The peak observed in the curves corresponding to standard INCL4 are of course due the absorption via the formation of a $\Delta$ resonance (followed by $\Delta N \rightarrow N N$ process) with free kinematics. A smoothing of the peak and a shift to lower energy are observed when $\pi$ potentials are introduced. The shift, particularly visible in light targets, may

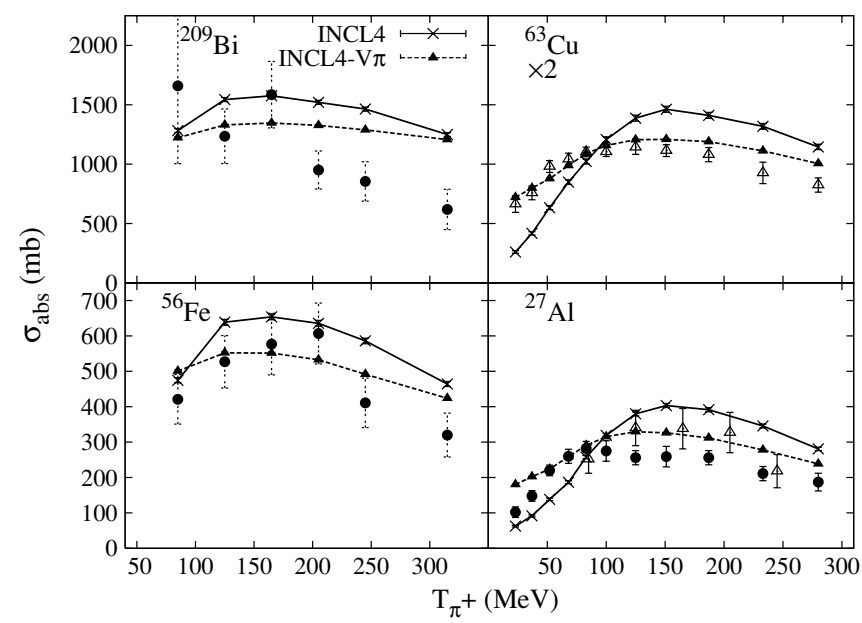

FIG. 15. Positive $\pi$ (true) absorption cross section on four different targets, indicated on the panels. Same convention as in Fig. 14. Data are from Refs. [25,26]. Note that all cross sections for ${ }^{63} \mathrm{Cu}$ have been multiplied by 2 .

be understood as a binding effect. This feature is reminescent of the peak observed in total $\pi N$ cross sections, for which the same explanation is often advanced. Note, however, that for heavy targets, we still overestimate noticeably the absorption cross section at high energy and underestimate it at low energy. The last defect is mainly visible for $\pi^{-}$'s. It may be related to an inherent shortcoming of our semiclassical simulation. Indeed, the de Broglie wavelength of low-energy $\pi$ 's is large and in addition, the attractive Coulomb interaction for the $\pi^{-}$ case may focalize the incoming wave and enhance strongly the absorption. Note, however, some inconsistency of the data for light targets.

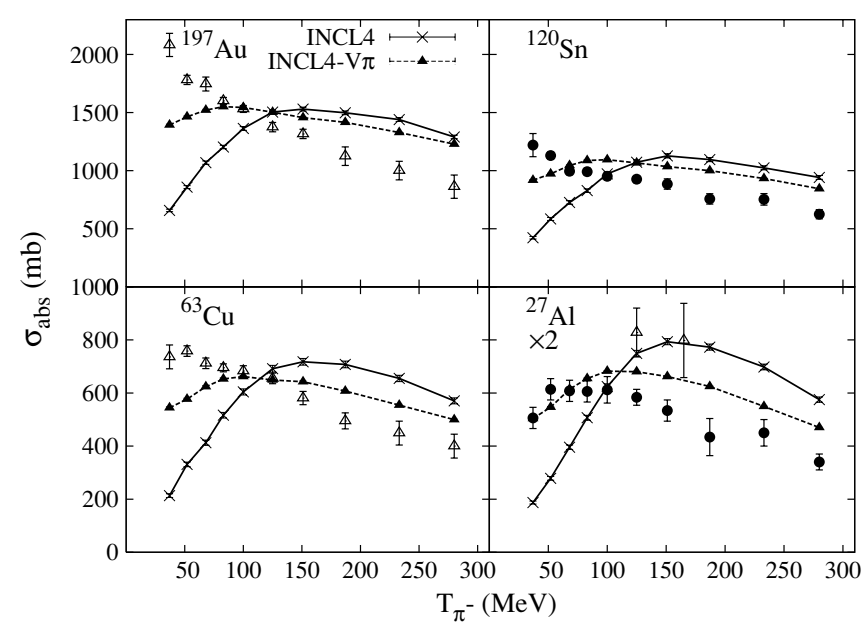

FIG. 16. Negative $\pi$ (true) absorption cross section on four different targets (indicated in the respective panels). Same convention as described in the legend to Fig. 15. Data are from Refs. [25,26]. Note that all cross sections for ${ }^{27} \mathrm{Al}$ have been multiplied by 2 . 
TABLE II. Comparison of calculated total cross sections (in mb) for production of protons with a kinetic energy larger than $40 \mathrm{MeV}$ in $\pi$-induced reactions, with the experimental values of Ref. [24], where experimental uncertainties are not quoted, for three different incident energies (in $\mathrm{MeV}$ ) and three different targets. For each target nucleus, we give the calculated results with standard INCL4 (second line) and with the modifed version (third line).

\begin{tabular}{llrrrrrr}
\hline \hline & & \multicolumn{3}{c}{$\pi^{+}$} & & $\pi^{-}$ \\
\hline$T_{\pi}$ & (in MeV) & 100 & 160 & 220 & 100 & 160 & 220 \\
${ }^{12} \mathrm{C}$ & $\sigma$ (exp.) & 240 & 370 & 310 & 44 & 71 & 80 \\
& $\sigma$ (calc.) & 205 & 391 & 458 & 48 & 93 & 109 \\
& $\sigma$ (calc.) & 199 & 350 & 373 & 48 & 81 & 92 \\
${ }^{n a t} \mathrm{Ni}$ & $\sigma$ (exp.) & 610 & 860 & 790 & 161 & 220 & 265 \\
& $\sigma$ (calc.) & 670 & 1037 & 1180 & 200 & 344 & 405 \\
& $\sigma$ (calc.) & 579 & 918 & 1036 & 184 & 287 & 351 \\
${ }^{181} \mathrm{Ta}$ & $\sigma$ (exp.) & 890 & 1280 & 1220 & 260 & 340 & 420 \\
& $\sigma$ (calc.) & 1148 & 1711 & 1985 & 372 & 581 & 720 \\
& $\sigma$ (calc.) & 1040 & 1659 & 1898 & 439 & 638 & 790 \\
\hline \hline & & & & & & &
\end{tabular}

\section{Proton and neutron production in pion-induced reactions}

Let us turn to proton emission in $\pi$-induced reactions. We compared our results with the measurements of Ref. [24]. In Table II, the total cross sections for production of protons of kinetic energy of more than $40 \mathrm{MeV}$ are given for three targets. Our predictions are improved by the introduction of the $\pi$ potential (except for $\pi^{-}$induced reactions on Ta), although they are not really satisfactory for the heaviest target (for both $\pi^{+}$- and $\pi^{-}$-induced reactions). The large difference observed in the cross sections for $\pi^{+}$and $\pi^{-}$is generally attributed to the differences of the yields corresponding to the conventional two-nucleon absorption mechanisms. According to Ref. [24],

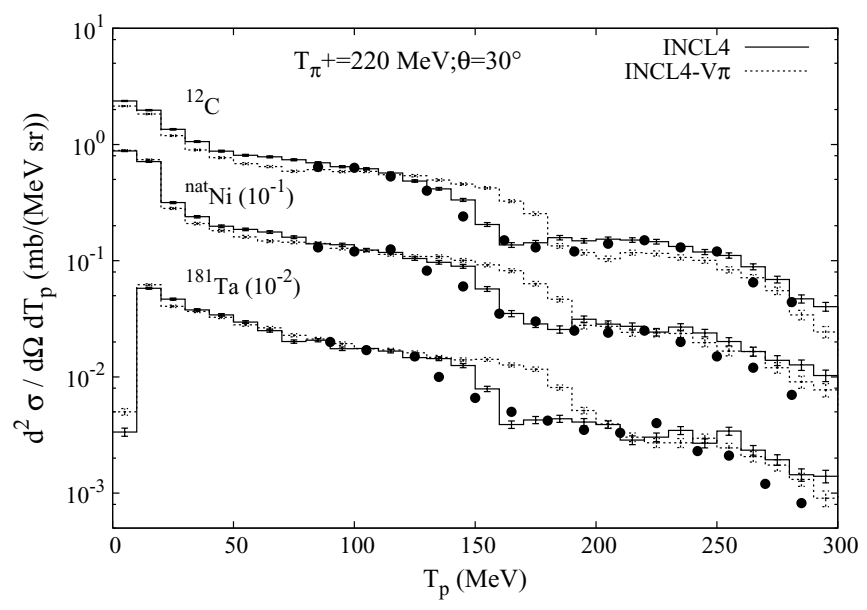

FIG. 17. Double differential cross section for proton emission at $30^{\circ}$ in $\pi^{+}$-induced reactions on three different targets at $220 \mathrm{MeV}$. To ease the reading of the figure, the successive spectra have been multiplied by decreasing powers of 10 , except for the lightest target, in which case absolute values are given. Data (dots) from Ref. [24] are compared with the standard predictions of INCL4 (full histograms) and with the predictions after the modifications described in the text (dotted histograms). The error bars give the typical uncertainty of the calculations.

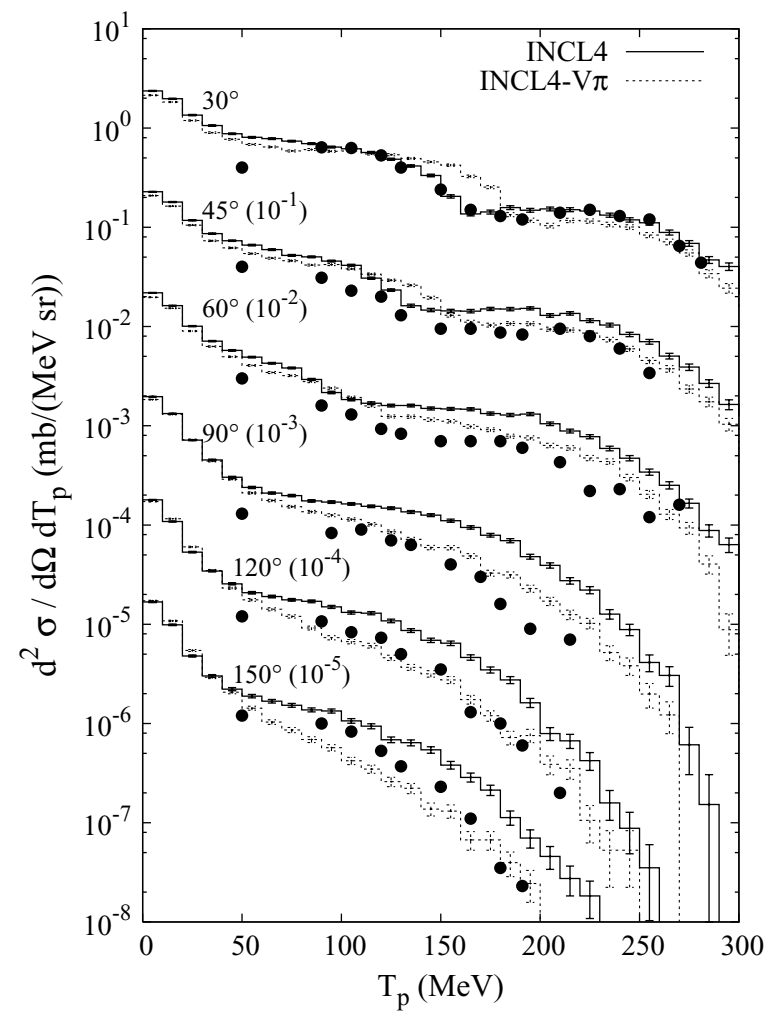

FIG. 18. Double differential cross section for proton emission in $\pi^{+}$-induced reactions on ${ }^{12} \mathrm{C}$ at $220 \mathrm{MeV}$. To ease the reading of the figure, the successive spectra have been multiplied by decreasing powers of 10, except for the smallest angle, in which case absolute values are given. Data (dots) from Ref. [24] are compared with the standard predictions of INCL4 (full histograms) and with the predictions after the modifications described in the text (dotted histograms). The error bars give the typical uncertainty of the calculations.

in the resonance region, the ratio of the proton yield for $\pi^{+}$'s to the one for $\pi^{-}$'s amounts to $\sim 13$ for ${ }^{12} \mathrm{C}$ and $\mathrm{Ni}$ and to $\sim 17$ for Ta. For the quasifree process $[\pi N$ collision through the $(3,3)$ resonance], the ratio is around 11 for all targts. The fact that both in experiment and in our calculations the ratios are well below the quoted values strongly indicates that protons are likely ejected in secondary collisions. The overestimate of the proton yield for the heavy target, in contrast with the reasonably good predictions for ${ }^{12} \mathrm{C}$ and $\mathrm{Ni}$, leads to believe that we overestimate secondary reactions.

Figure 17 shows a comparison of calculated and experimental double differential cross sections at $30^{\circ}$, for $\pi^{+}$-induced reactions on the same targets. Our results compare reasonably well with the experimental data. However, in this case, the introduction of the $\pi$ potential slightly worsens the results. This is somewhat annoying. The two shoulders correspond roughly to proton production by direct $\pi N$ scattering, which should be prominent around $90 \mathrm{MeV}$, and to production by the $\Delta N \rightarrow N N$, which should dominate around $250 \mathrm{MeV}$. The fact that the first shoulder is shifted to higher energy seems to indicate that the $\pi^{+}$potential introduced is too large and that no potential would be a better choice. However, one has to keep in mind that the "width" of the shoulders are given by 


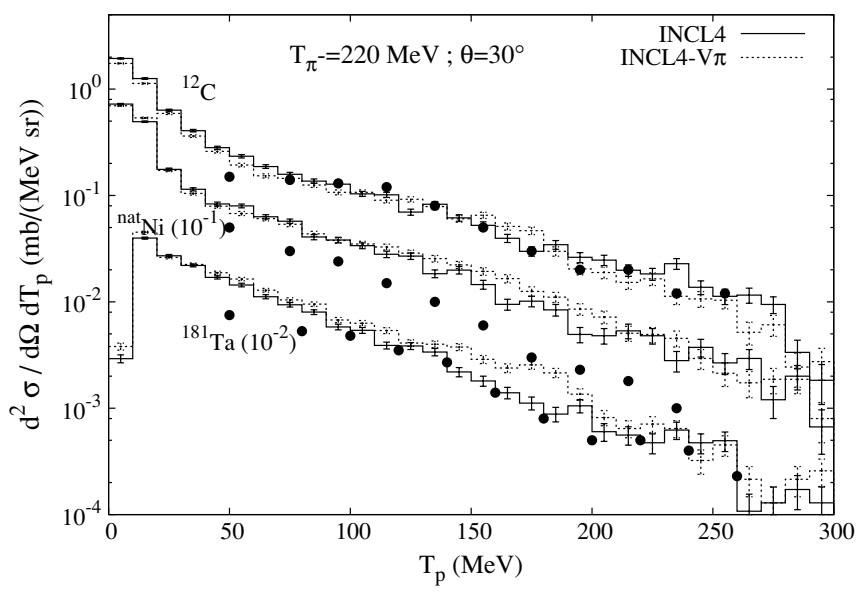

FIG. 19. Same as described in the legend to Fig. 17 for negative $\pi$ 's. Data are from Ref. [24].

Fermi motion and that the protons are produced not only by the first interaction but also by secondary ones, which correspond to different kinematics. Therefore, it is perhaps not wise to draw definite conclusion from this picture. Figure 18 shows the same comparison for all angles in the case of the $\mathrm{C}$ target. If the same defect as in the previous figure is also to be seen for the first two angles, the introduction of $\pi$ potential definitely improves the description of the data, especially in the backward hemisphere. Figure 19 shows the same comparison as Fig. 17, but for $\pi^{-}$-induced reactions. In this case, the defect observed previously is less marked and the results are rather satisfactory for the light and the heavy targets but surprisingly overestimate the cross section in the case of the intermediate target.

In Fig. 20, we display the results with our modified version for neutron production in $\pi^{+}$-induced reactions at $870 \mathrm{MeV}$. The overall agreement is rather satisfactory. The more or less correct normalization comes from our use of a realistic $\pi-N$ cross section at high energy (see Appendix). A closer look at the figure indicates that the high-energy part of the spectra is overestimated at intermediate angles and that the evaporation component is slightly overestimated. We have no clear explanation for these deficiencies. However, the presence of a peak at $15^{\circ}$ and high neutron energy (due to the $\pi N \rightarrow \Delta, N \Delta \rightarrow N N$ mechanism) is roughly reproduced by our calculations. We did not compare here with INCL4 predictions, because this model does not have a good $\pi-N$ cross section at the $\pi$ incident energy, as we indicated in Sec. III (see also the Appendix).

\section{Residue production in pion-induced reactions}

Experimental data concerning residue production are very scarce. We compared our results for $\pi$-induced reactions on a natural $U$ target at $80 \mathrm{MeV}$ with the experimental data of Ref. [30]. The total fission cross sections are given in Table III. These quantities mainly test the fission model, but they also partially test the INC model that determines the excitation energy of the fissioning remnant. Clearly, the results are improved by our modifications, presumably due to the increase of the excitation energy. Our results are satisfactory

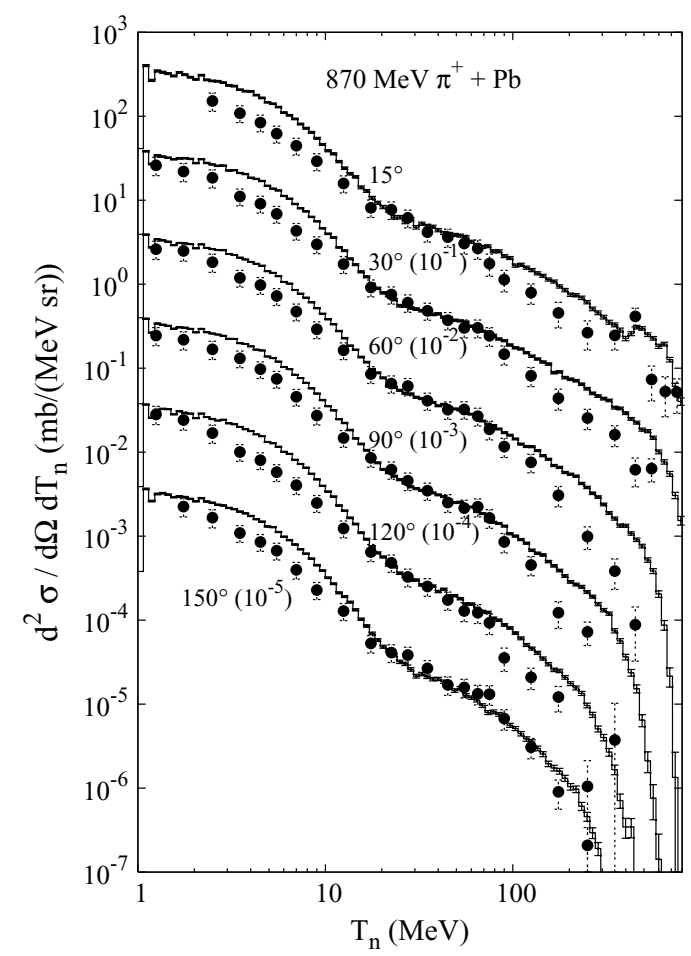

FIG. 20. Neutron double differential cross section in $\pi^{+}-\mathrm{Pb}$ collisions at $870 \mathrm{MeV}$. Data from Ref. [29] (dots) are compared with the calculations (histograms) using our modified version.

for $\pi^{+}$reactions but underpredict the fission cross section for $\pi^{-}$reactions. This is a rather puzzling situation. Total reaction cross sections (extrapolated from the $\mathrm{Bi}$ value and the trend indicated in Ref. [25]) are about the same for $\pi^{+}$or $\pi^{-}$: about $3000 \mathrm{mb}$. The experimental fission cross section is about $2 / 3$ of this value for $\pi^{+}$but exhausts the total cross section for $\pi^{-}$. This seems to indicate that the fission mechanism might be different for the two kinds of $\pi$ 's. True absorption, which we reproduce rather well in this energy range, for both cases (see Figs. 15 and 16), does not seem to play an important role: the cross section is larger for the $\pi^{+}$case than for the $\pi^{-}$one.

In Fig. 21 we show the mass yield of fission fragments, along with our predictions using INCL4 coupled to two evaporation-fission models, namely the version KHSv3p [6] of the ABLA model developed by K.-H. Schmidt [4,5] and the GEM model $[31,32]$. Both models correctly reproduces the

TABLE III. Total fission cross section (in $\mathrm{mb}$ ) in pion-induced reactions on $\mathrm{U}$ at $80 \mathrm{MeV}$. Predictions (third and fourth columns) are given for INCL4 coupled to the evaporation-fission model of K.-H. Schmidt [4,5] (version KHSv3p [6]). For both kind of $\pi$ 's, we give the predictions of the standard INCL4 and those with our modified version. Data are from Ref. [30].

\begin{tabular}{lccc}
\hline \hline \multirow{3}{*}{ Experiment } & \multicolumn{2}{c}{ Theory } \\
\cline { 3 - 4 } & & INCL4(standard) & INCL4(mod.) \\
\hline$\pi^{+}$ & $2031 \pm 45$ & 1681 & 1941 \\
$\pi^{-}$ & $2905 \pm 58$ & 1480 & 1947 \\
\hline \hline
\end{tabular}


TABLE IV. Calculated average excitation energy at the end of the cascade phase (in $\mathrm{MeV}$, second column), proton multiplicity (third column), and neutron multiplicity. The latter is split in three energy domains. For each reaction system indicated in the first column, the first line corresponds to the standard INCL4 model and the second line gives our predictions with the modified version.

\begin{tabular}{lccccc}
\hline \hline & $\left\langle E^{\star}\right\rangle(\mathrm{MeV})$ & $p$ & \multicolumn{3}{c}{$\mathrm{n}$} \\
\cline { 4 - 5 } & & & $T_{n}<2 \mathrm{MeV}$ & $2<T_{n}<20 \mathrm{MeV}$ & $T_{n}>20 \mathrm{MeV}$ \\
\hline$p(800 \mathrm{MeV})+\mathrm{Pb}$ & 120.3 & 2.57 & 3.33 & 6.83 & 2.48 \\
& 136.3 & 2.74 & 3.47 & 7.42 & 2.22 \\
$p(1200 \mathrm{MeV})+\mathrm{Pb}$ & 148.2 & 3.23 & 3.39 & 8.11 & 3.18 \\
& 175.5 & 3.53 & 3.56 & 9.13 & 2.94 \\
$p(1600 \mathrm{MeV})+\mathrm{Pb}$ & 165.7 & 3.69 & 3.41 & 8.78 & 3.66 \\
& 202.1 & 4.16 & 3.57 & 10.12 & 3.48 \\
$p(800 \mathrm{MeV})+\mathrm{Fe}$ & 69.6 & 3.49 & 0.59 & 1.97 & 1.64 \\
& 85.5 & 3.61 & 0.61 & 2.20 & 1.57 \\
$p(1200 \mathrm{MeV})+\mathrm{Fe}$ & 78.9 & 3.85 & 0.59 & 2.15 & 1.95 \\
& 99.5 & 4.09 & 0.62 & 2.47 & 1.93 \\
$p(1600 \mathrm{MeV})+\mathrm{Fe}$ & 84.2 & 4.06 & 0.60 & 2.24 & 2.14 \\
& 107.5 & 4.35 & 0.62 & 2.59 & 2.13 \\
\hline \hline
\end{tabular}

width of the mass yield distribution, which is sizably larger than for low-energy neutron-induced fission. This is mainly attributable not only to the fission models but also to the predicted excitation energy. The shape of the mass distribution differs in the two models, which points to a different energy dependence of the fission probability in the two models. To fix the ideas, let us mention that the calculated excitation energy lies around $80 \mathrm{MeV}$, on the average. Figure 21 refers to $\pi^{+}$-induced fission, but very similar results are obtained for $\pi^{-}$-induced fission, whose data are also reported in Ref. [30].

It is also interesting to compare our predictions with the data of Ref. [33] for fission cross section in $\pi^{+}$-induced fission (see Fig. 22). One can see that we globally reproduce the trend, except for $\mathrm{U}$ above $150 \mathrm{MeV}$. However, one has to realize these experimental points, when compared with a reasonable value of the reaction cross section yield a fission probability larger than 1 [33]. Our modified version slightly improves on the standard INCL4 results and gives similar results to the CEM model of Ref. [34], as reported in Ref. [33]. The influence of the $(3,3)$ resonance is barely visible on the energy dependence of the fission cross sections.

\section{E. More sophisticated pion potentials}

We argued in Sec. IV that the value of the potential inside the nuclear volume is somewhat irrelevant. To check this assertion, we decided to test two potentials that embody some features of the behavior of the real part of the optical-model potential as depicted in Fig. 4. In one case, we reversed the sign of the (nuclear) potential for $r<r_{1}$, with $r_{1} \approx R_{0}-2 \mathrm{fm}$, introducing this way some repulsive core. In the other case, we introduced an energy dependence on $V_{N}^{0}$, which is approximately $-30 \mathrm{MeV}$ for kinetic energy below $100 \mathrm{MeV}$ and that raises progressively to $20 \mathrm{MeV}$ to around $350 \mathrm{MeV}$. In both cases, the effect remains weak. For instance, concerning $\pi$ double differential cross sections (see Figs. 10-13), the effect is roughly of the order of the error bars of the calculations.

\section{F. Influence of the $\Delta$ mass}

We investigated the influence on our results of the lowering of the $\Delta$ mass, by $50 \mathrm{MeV}$ at the most. It seems that the results are largely insensitive to such a modification, except for the absorption cross section at low energy (for less than $50 \mathrm{MeV}$ ): the cross sections are substantially increased, removing the discrepancy observed for negative $\pi$ 's in this energy range (Fig. 16) but overestimating the cross section for positive $\pi$ 's in the same energy range (Fig. 15).

\section{G. Indirect influence of the modification of the pion scenario}

We here pay attention to quantities that do not focus on $\pi$ 's in the entrance or in the final channels and are thereby only influenced indirectly by a modification of the $\pi$ production mechanism. The most obvious one is the neutron (or proton) production cross section in proton-induced reactions. In Table IV, we give the neutron multiplicities in a typical case. We see that the multiplicity of the "cascade" neutrons (more precisely neutrons with a kinetic energy larger than $20 \mathrm{MeV}$ ) decreases when going from the standard to the modified versions. On the contrary, the proton multiplicity (basically emitted in the cascade) increases. In fact this trend is related not so much to the modification of the $\pi$ sector but rather to the introduction of isospin and energy-dependent potentials for the baryons, as explained in Ref. [8] (note that the study in this reference is based on the INCL3 version and not INCL4, but the conclusions are basically the same). The modification of the $\pi$ scenario typically accounts for $\sim 25 \%$ of the changes in the particle multiplicities referred above. Table IV also shows that there is an enhancement of the evaporated neutrons. This is correlated with the increase of the excitation energy, indicated in the same table. The origin of this change is simply due to the lesser number of emitted $\pi$ 's.

To avoid any confusion, let us make some comment on the last result, in comparison with the discussion of Sec. II. 


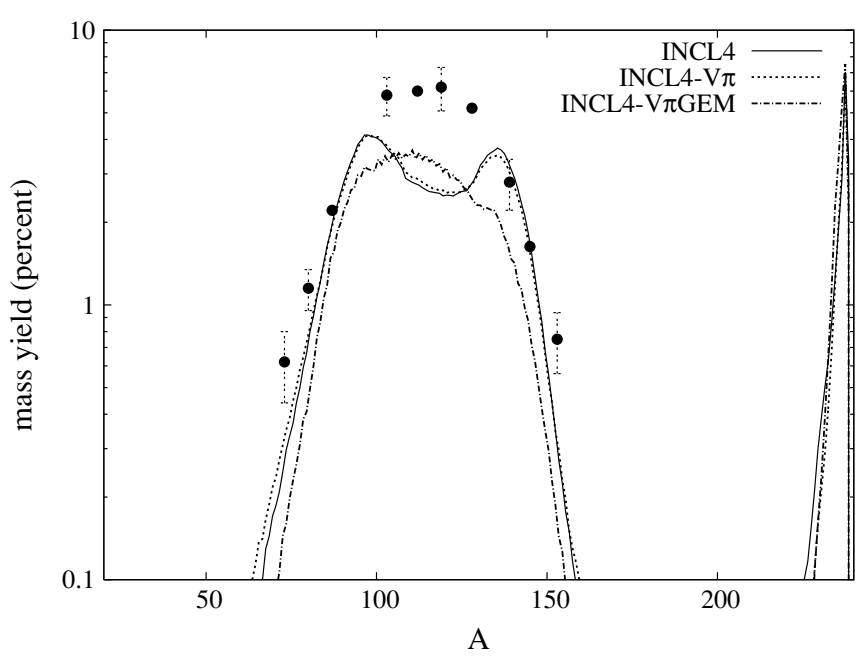

FIG. 21. Mass yield of the fission fragments in $\pi^{+}$-induced reactions on ${ }^{\text {nat }} \mathrm{U}$ at $80 \mathrm{MeV}$. The yield is defined as the ratio between the production cross section for a given fragment and the fission cross section. The dots are the data from Ref. [30], reproduced as in the publication. However, they sum up to $\sim 300 \%$ instead of $200 \%$ for binary fission. The lines correspond to the yields calculated with the standard INCL4 (full line), with our modified version coupled to the ABLA evaporation-fission model [4,5] (dotted line) or to the GEM model [31,32].

When we cut the inelastic channel, keeping the same $N N$ total cross section, $\pi$ production is evidently (totally) reduced, in comparison with the standard case, and neutron production is also reduced. When we change the $\pi$ production scenario, as described above, $\pi$ production is also (partially) reduced and the neutron yield is enhanced. The difference in the behavior of the neutron yield (mainly coming from evaporation) is explained as follows. In the first case, the inelasticity of $N N$ collisions is reduced, less energy is transferred to the nucleus, the excitation energy at the end of the cascade is reduced, and fewer neutrons are emitted. In the second case, the inelasticity is kept the same, basically the same energy is transferred, but the modifications of the $\pi$ scenario (mainly the $\pi$ potential) introduce somewhat less transparency for the $\pi$ 's. As a result, fewer $\pi$ 's are emitted, the excitation energy is increased, and more neutrons are produced.

In Fig. 23, we compare our predictions for the residue mass and charge spectra, with and without modification of the $\pi$ scenario. We see that the so-called spallation peak is broadened. This improvement is obtained at the expense of a slight decrease of the cross section for residues close to the projectile. This change is, of course, due to the increase of the excitation energy left after the cascade stage. The latter statement is strongly supported by Fig. 24, which displays the distribution of the excitation energy. The shape of the isotopic distributions (not shown) is hardly modified.

\section{DISCUSSION}

\section{A. Introduction}

Describing $\pi$ aspects of spallation reactions by semiclassical tools as in our approach is certainly less founded than for

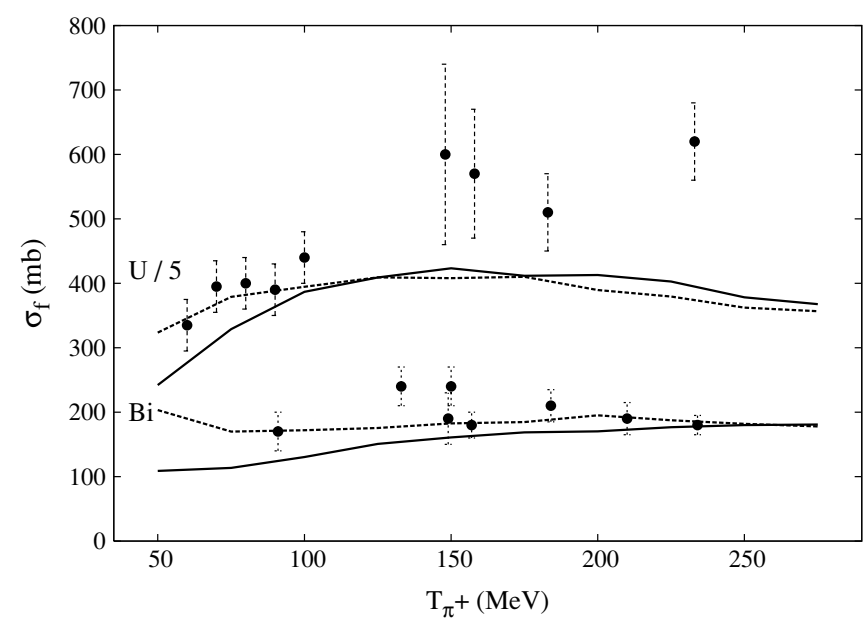

FIG. 22. Fission cross section in $\pi^{+}$-induced reactions on ${ }^{235} \mathrm{U}$ and ${ }^{209} \mathrm{Bi}$, as a function of the incident pion energy. The symbols represent the data from Ref. [33]. The full lines correspond to the yields calculated with the standard INCL4. The dotted lines correspond to our modified version, coupled to the ABLA evaporation-fission model $[4,5]$. Note the $U$ cross sections have been divided by 5 .

nucleons. Furthermore, absorption of low-energy $\pi$ 's involves at least two nucleons in a way that is probably not reducible to our scenario. In spite of these difficulties, it seems that our treatment has the virtue of mocking up correctly the main features of $\pi$ production. A wise and often advocated procedure is to test the model on the $\pi$-induced reactions. That is what we did, with some (limited) success, in this article. To assess the capabilities of our model, we may also compare with other (quantum) theoretical models, which have been used for describing $\pi N$ interactions. There are basically two kinds of such theoretical approaches. One is based on the multiple-scattering theory [36,37] and includes $N$ and $\pi$ degrees of freedom. Most of its applications use factorization and fixed-scatterer approximation (see Ref. [19] for a review). Because the $\Delta$ degrees of freedom are neglected, this approach is suited to describe the $\pi$ optical model and excitations to low-lying states (through DWBA). The Kisslinger potential can be derived from this approach when the $s$-and $p$-wave nature of the $\pi N$ interaction is made explicit. The other kind of approach is the so-called $\Delta$-hole model [38-42], which includes $\Delta$ degrees of freedom. It is then more appropriate for $\pi$ 's in the $\sim 80$ - to $300-\mathrm{MeV}$ domain. This model can handle true absorption, as recalled below. We thus preferred to make a (short) comparison with this model. It should, however, be stressed that our model can make predictions for more complicated processes, such as the generation of a specific residue after removal of several nucleons.

\section{B. Comparison with the $\Delta$-hole model}

Just to make the discussion more transparent, we review quickly the main features of the $\Delta$-hole model. In this model, the available space is divided into three subspaces: $P$, the product of the $\pi$ space and of the target eigenstates; $D$, the product of the $\Delta$ space and the eigenstates of the nucleus 

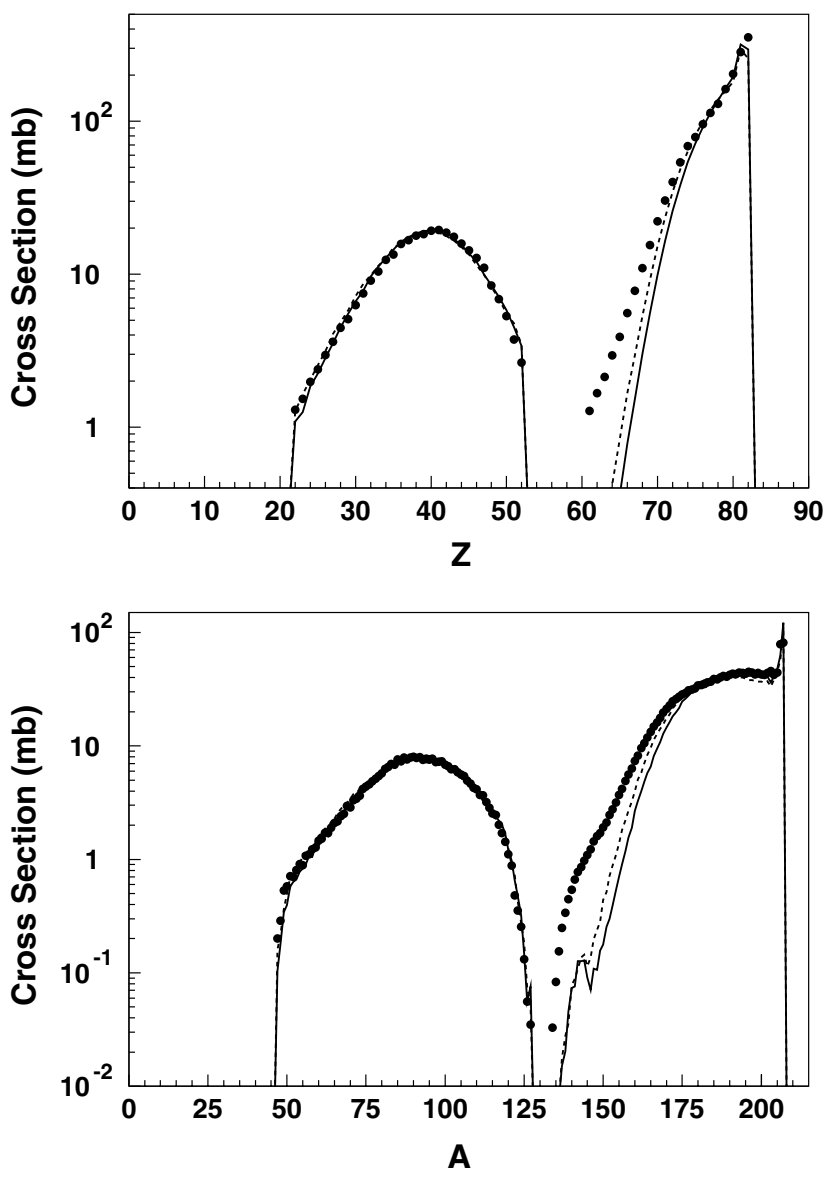

FIG. 23. Residue charge (upper panel) and mass (lower panel) production cross sections in $p$ - $\mathrm{Pb}$ collisions at $1 \mathrm{GeV}$. Data (dots) from Ref. [35] are compared with our calculations using the standard (full lines) and the modified (dotted lines) versions. Only calculations for measured residues are displayed.

obtained by removing a nucleon (hole) from the target; and $Q$, the space of the eigenstates of the target alone. The Hamiltonians acting in these subspaces are

$$
\begin{aligned}
& H_{P}=H_{A}+T_{\pi}+V_{\pi A}, \\
& H_{D}=H_{A-1}+T_{\Delta}+V_{\Delta},
\end{aligned}
$$

and

$$
H_{Q}=H_{A},
$$

respectively. In these equations, $H_{A}$ is the Hamiltonian of nucleus of mass $A, V_{\pi A}$ is the interaction of the $\pi$ with the nucleons, and $V_{\Delta}$ is the interaction of the $\Delta$ with the remaining nucleons. The $\Delta$-hole model assumes that there is no direct connection between the $P$ and $Q$ subspaces. Transition between the $P$ and $D$ spaces is generated by a $\pi N \rightleftharpoons \Delta$ vertex $f_{\pi N \Delta}$. By using standard projection techniques, the scattering operator may be written as

$$
T(E)=T_{\mathrm{nr}}(E)+T_{r}(E)
$$

where the nonresonant part $T_{\mathrm{nr}}(E)$ is due to the nonresonant interaction $V_{\pi A}$ in $P$ space, and the resonant part $T_{r}(E)$ can be

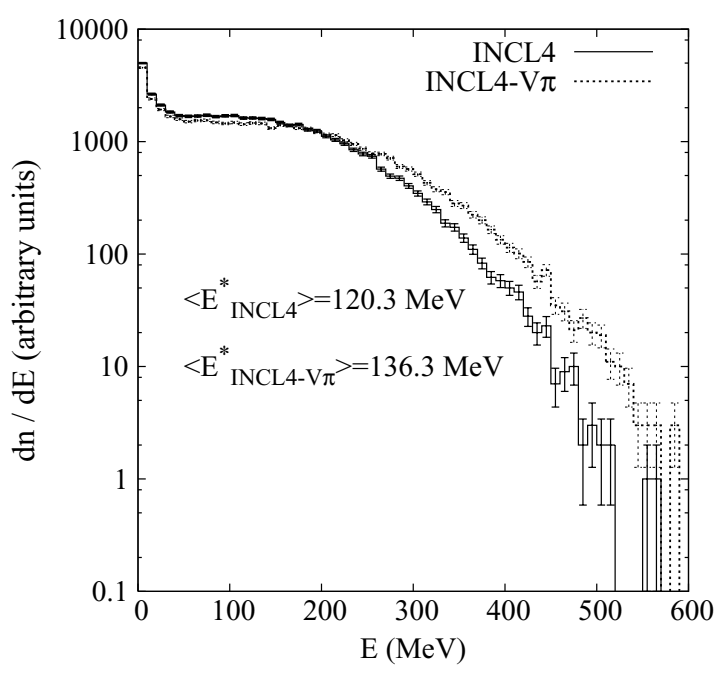

FIG. 24. Distribution of the excitation energy $E^{\star}$ left in the remnant at the end of the cascade stage. Comparison between the results obtained with the standard version of INCL4 (solid histogram) and those obtained with the modified version (dashed histogram). The figure refers to the $p(800 \mathrm{MeV})+{ }^{208} \mathrm{~Pb}$ case.

written as:

$$
T_{r}(E)=\frac{\Omega_{\mathrm{nr}}^{(-) \dagger}(E) f_{\pi N \Delta}^{\dagger} f_{\pi N \Delta} \Omega_{\mathrm{nr}}^{(+)}(E)}{E-H_{D}-\Sigma_{\text {free }}-W^{\uparrow}-\Sigma_{\text {Pauli }}-W_{\mathrm{sp}}} .
$$

In this equation, the Møller operators $\Omega$ introduce distorted waves due to the nonresonant scattering, $\Sigma_{\text {free }}$ is the free $\Delta$ propagator, $W^{\uparrow}$ is due to the interaction of the $\Delta$ with the target in its ground state, and $\Sigma_{\text {Pauli }}$ corrects the preceeding term by forbidding occupied single-particle states. Finally, $W_{\text {sp }}$ accounts for the coupling to $Q$ space and allows for $\pi$ absorption effects via the $\Delta N \rightleftharpoons N N$ process. The last four terms have real and imaginary parts. There have been a lot of investigations concerning the real part of $W^{\uparrow}$, which can be influenced by the residual interaction through the so-called spin-isospin Landau parameter [18]. The imaginary part of $W^{\uparrow}$ slightly increases the $\Delta$ width, the one of $-\Sigma_{\text {Pauli }}$ decreases it (as it corrects for forbidden transitions). Finally the imaginary part of $W_{\mathrm{sp}}$ introduces a collisional broadening of the $\Delta$ resonance. With a bit of phenomenology, the $\Delta$-hole model reaches a satisfactory agreement with experiment for elastic and absorption cross sections. It is much less successful for inclusive inelastic scattering. It has not been really applied for proton production or for residue cross sections. A comparison between our results and those of the $\Delta$-hole model contained in Ref. [43] is given in Figs. 25 and 26. One can see that our results are of a quality comparable with the one achieved with the $\Delta$-hole model. Sometimes they are even better, but, on the other hand, we overestimate the absorption cross section above the $(3,3)$ resonance. It is very hard to trace back the origin of the differences to the ingredients of the respective models. We come back to this point in the next subsection. 


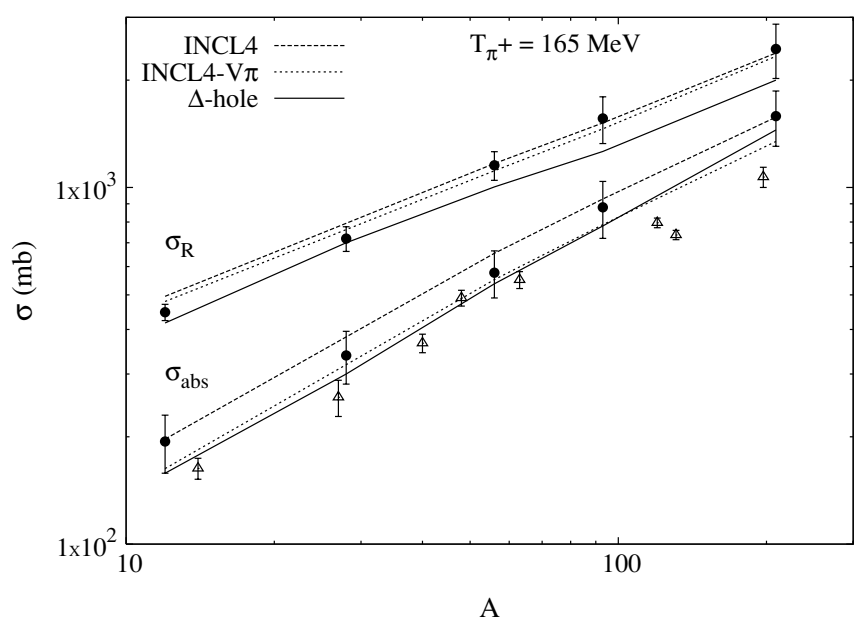

FIG. 25. Reaction and true absorption cross sections for $165-\mathrm{MeV}$ positive pions on various targets. Comparison between the predictions of standard INCL4 (dashed lines), of the modified version (dotted lines), of the $\Delta$-hole model of Ref. [43] (full lines), and the experimental data (symbols) of Refs. [25,26]

\section{Discussion of our results}

Our results, which compare favorably with experimental data, though not perfectly, raise an important question: is a multiple scattering quasiclassical model suitable for the description of $\pi$ degrees of freedom in hadron-nucleus collision in the energy range under consideration here? The conditions of validity of the intranuclear cascade, which demand that the de Broglie wavelength for the relative motion to be much smaller than the interaction range, which in turn should be smaller than the average distance between neighboring particles, are barely fulfilled for nucleons. Yet, the intranuclear cascade seems to work well, even at low incident energy [44]. Of course, as we said above, we are in an even less favorable case for $\pi$ 's: for the same kinetic energy, the de Broglie wavelength is larger for $\pi$ 's than for nucleons. Hence, quantum motion effects are expected to leave some fingerprint. Furthermore, the interaction of a low-energy $\pi$ with two nucleons may not be reducible to a succession of two single-nucleon interactions. In our opinion, this could explain why we underestimate the (negative) $\pi$ absorption cross section at low energy. This may also be at the origin of the overestimate of low energy $\pi^{+}$emission in proton-induced reactions (see Fig. 9), although it is then hard to explain that we predict correctly the $\pi^{-}$emission. The conclusion is that, even when disregarding obvious quantum motion (wave packet) features, we arrive nevertheless at globally satisfactory results. This presumably means that our simulation mocks up these features efficiently, on the average.

A way to test our modeling of the $\pi$ degrees of freedom is to look at our predictions of $\pi$-induced reactions. We, of course, cannot describe elastic scattering (which is of minor importance for spallation sources). However, we can reproduce rather well reaction cross sections (except on heavy targets for low energy $\pi^{-}$'s). In some sense, this is not surprising, as one is not far from the strong absorption limit (in the elastic channel) and it is sufficient to have a large $\pi N$ cross section.

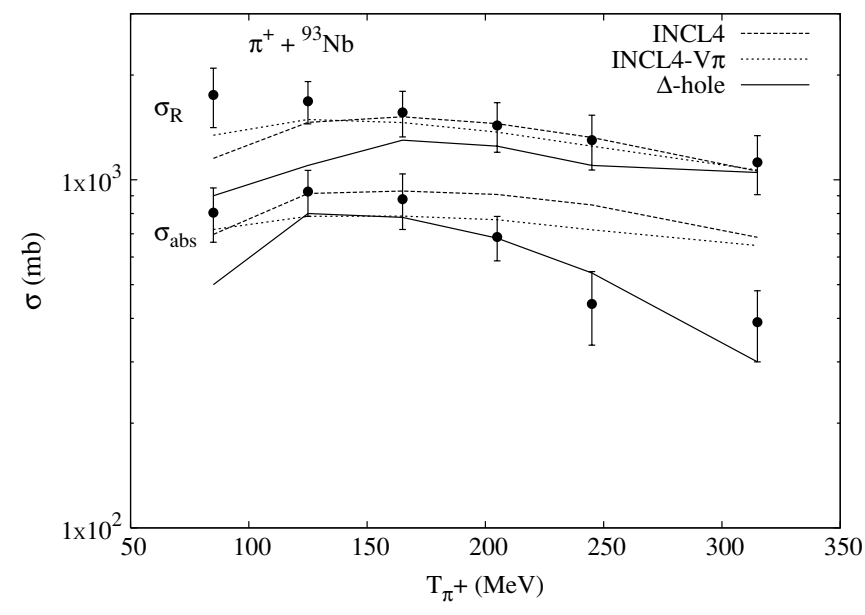

FIG. 26. Reaction and true absorption cross sections for positive $\pi$ 's on a ${ }^{93} \mathrm{Nb}$ target, as a function of the incident kinetic energy. Comparison between the predictions of standard INCL4 (dashed lines), of the modified version (dotted lines), of the $\Delta$-hole model of Ref. [43] (full lines), and the experimental data (dots) of Ref [25].

It is nevertheless interesting to see that we can reproduce some detail of these cross sections, as the dominance of the $\Delta$ resonance in light targets and the progressive shift of the maximum to lower incident energy as the target mass decreases (see Fig. 14). This reflects the importance of secondary collisions and the fact that we probably picked up the main features of the $\pi$-nucleus mechanism. True absorption cross sections provide with a more stringent test, as they account only for about one half of the reaction cross sections. Again, the fact that they are reproduced rather well in our calculations (see Figs. 15-16) indicates that the two-step mechanism is largely dominant. More interestingly, the introduction of the $\pi$ potential improves on our description by INCL4. This points to binding effects in true absorption and probably also indicates that our $\pi$ potential is realistic.

An interesting test of the $\pi$ dynamics would be provided by inelastic (and charge exchange) scattering of $\pi$ 's by nuclei. We do not include such a study in the present work, because data are rather scarce and sometimes imprecise [19]. Let us quote, however, the data mentioned in Ref. [45], which pertain to high-energy incident $\pi$ 's and which are reasonably reproduced by Mashnik's CEM model [46], provided $\pi N$ interaction is hindered for a small time span right after every $\pi N$ collision. We delay the comparison with this work to a future work for three reasons: (i) the $\pi$ energy range is somehow outside the one of general interest here, (ii) it is not clear that the hindrance of $\pi N$ interaction introduced for $\pi$ inelastic scattering is not harmful for the description of other data, (iii) the approach of Ref. [46] is a combination of intranuclear cascade and exciton model; the relevance of this preequilibrium stage, which is avoided in INCL4, is still a question under debate $[6,47]$.

Pion production in proton-induced reactions (our main concern) and proton production in $\pi$-induced reactions are more difficult to analyze. In our description, $\pi$ 's can be produced at any time and very likely at any place in the intranuclear cascade. At $700 \mathrm{MeV}$ and above, the incident proton can produce a $\pi$ in the first collision with a probability 


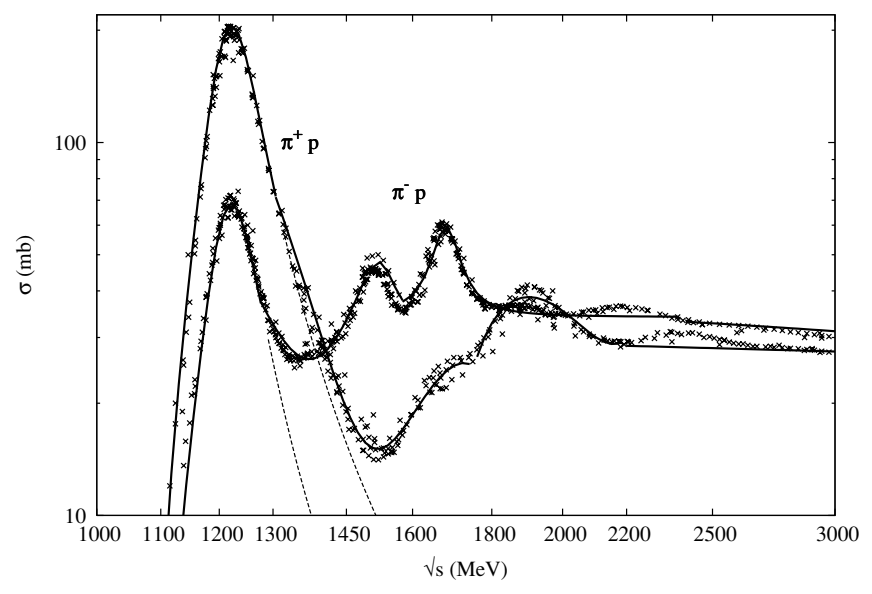

FIG. 27. Fits of $\pi^{+}-p$ and $\pi^{-}-p$ total cross sections. The crosses are the Particle Data Group world experimental data [48]. The full lines correspond to the parametrizations used in our modified version. The dotted lines [equivalent to the full lines in the $(3,3)$ resonance region] give the parametrization used in the standard INCL4 model.

of one-half. However, except for peripheral collisions, this $\pi$ is reabsorbed and an other one is produced (several times) before final $\pi$ 's are emitted. In other words, outgoing $\pi$ 's are produced near the surface. This is presumably the reason why our results are somehow insensitive to the $\pi$ potential in the nuclear interior (note that this is not the same reason why optical-model fits, which pertains to the description of the incoming $\pi$ only, are insensitive to the same quantity). However, what matters is the $\pi$ potential in the surface region, the transmission to the Coulomb barrier and, of course, the flux of energetic nucleons near the surface. The remarkable improvement of our results after introduction of $\pi$ potentials (nuclear+Coulomb) is also a justification of the appropriateness of our potentials, at least for our simulations.

\section{CONCLUSION}

In this article, we have reexamined the $\pi$ sector of the INCL4 model for spallation reactions. The latter presented some deficiencies, in particular a sizable overestimation of the $\pi$ yield in proton-induced reactions. We put here the emphasis on the proper $\pi N$ cross section above the $(3,3)$ resonance and to the introduction of $\pi$ potentials neglected so far. Disregarding largely the information from the phenomenology of the $\pi$-nucleus optical potential, mainly because it is basically determined by the strong absorption of the flux in the elastic channel and therefore poorly correlated to the energy of $\pi$ excitation inside the nuclear volume, we adopted a pragmatic approach, introducing a simple $\pi$ potential and determining its strength by a rough fitting on data, including $\pi$ production in proton-induced reactions but also $\pi N$ reactions. It is rewarding that the potentials so determined are consistent with the phenomenological real part of the $\pi$ optical-model potentials in the surface region. We also checked that, for the same kind of data, the value of the $\pi$ potential in the nuclear volume has a minor effect.
Introducing the modifications described above in INCL4 considerably improves our predictions in the $\pi$ sector and generates a globally rather satisfactory description of $\pi$-induced reactions. It also slightly improves our predictions for nucleon and residue production.

However, sometimes, noticeable shortcomings are still present. The most important one is the underprediction of the true $\pi$ absorption cross section at low energy and the small overprediction of this quantity above the $(3,3)$ resonance, mainly for negative $\pi$. The dominance of this resonance in the absorption cross section for light targets (and to some extent in reaction cross sections) suggests than absorption proceeds predominantly by the formation of the $\Delta$ resonance and the subsequent reinteraction of the latter with other nucleons, like in our model and many other theoretical investigations. The lack of $\pi$ absorption at low energy in our model may result partly from our neglect of $\pi N S$-wave interaction and from the fact that our quasiclassical method cannot cope with wave function effects at low energy. If the latter feature is the most important one, this deficiency might not be curable in our approach. Another source for the above-mentioned deficiency could arise from an unsatisfactory treatment of the $\Delta$ degrees of freedom. We assumed here that the $\Delta$ interactions can be treated by cross sections and are basically as in free space. There are indications that these interactions might be changed strongly in the nuclear medium, although theoretical guides are rather vague. That is why we did not investigate this possiblity (except for the average mass of the $\Delta$ ), even phenomenologically. This perhaps warrants a further investigation.

\section{ACKNOWLEDGMENTS}

We are very grateful to Drs. A. Boudard and S. Leray for interesting discussions. We also acknowledge the help of Dr. A. Boudard for the determination of the stopping time for $\pi$-induced cascades. This work has been performed within the framework of the EU IP EUROTRANS project (European Union contract FI6W-CT-2004-516520). We acknowledge the EU for financial support.

\section{APPENDIX: PARAMETRIZATION OF THE $\pi$-N TOTAL CROSS SECTION}

Figure 27 shows the experimental data for $\pi N$ total cross sections [48], along with the parametrizations used in standard INCL4 [6] and the fits used in our modified version. For $\pi^{0}$, the mean of these two cross sections is used. To attempt to account for many-body reactions at very low energy, a cross section of $5 \mathrm{mb}$ is imposed for all $\pi$. by

The parametrization used for $\pi^{+}$proton is given explicitly

$$
\sigma=\frac{326.5 q^{3}}{\left[1+4\left(\frac{\sqrt{s}-1215}{110}\right)^{2}\right]\left(q^{3}+180^{3}\right)}
$$


for $\sqrt{s}<1290 \mathrm{MeV}$,

$$
\begin{aligned}
\sigma= & -2.337 \times 10^{-6} s^{3 / 2}+1.138 \\
& \times 10^{-2} s-18.40 s^{1 / 2}+9893
\end{aligned}
$$

for $1290 \mathrm{MeV}<\sqrt{s}<1750 \mathrm{MeV}$,

$$
\begin{aligned}
\sigma= & 1.135 \times 10^{-6} s^{3 / 2}-6.917 \\
& \times 10^{-3} s+13.99 s^{1 / 2}-9361,
\end{aligned}
$$

for $1750 \mathrm{MeV}<\sqrt{s}<2175 \mathrm{MeV}$, and

$$
\sigma=-3.181 \ln (\sqrt{s})+52.978
$$

for $\sqrt{s}>2175 \mathrm{MeV}$. The quantity $q$ is the center-of-mass momentum

$$
q=\sqrt{\frac{\left(s-\left(m_{N}+m_{\pi}\right)^{2}\right)\left(s-\left(m_{N}-m_{\pi}\right)^{2}\right)}{4 s}} .
$$

Cross sections are in $\mathrm{mb}, \sqrt{s}$ in $\mathrm{MeV}$, and $q$ in $\mathrm{MeV} / c$.
For $\sqrt{s}<1290 \mathrm{MeV}$, the $\pi^{-} p$ cross section is taken as one-third of the $\pi^{+}-p$ one. Otherwise it is given by

$$
\sigma=1.2 \times 10^{-3}(\sqrt{s}-1372.52)^{2}+26.1058
$$

for $1290 \mathrm{MeV}<\sqrt{s}<1475 \mathrm{MeV}$,

$$
\begin{aligned}
\sigma= & 1.16 \times 10^{-5} s \\
& +49965.6 /\left[(\sqrt{s}-1519.59)^{2}+2372.55\right],
\end{aligned}
$$

for $1475 \mathrm{MeV}<\sqrt{s}<1565 \mathrm{MeV}$,

$$
\sigma=34.0248+43262.2 /\left[(\sqrt{s}-1681.65)^{2}+1689.35\right],
$$

for $1565 \mathrm{MeV}<\sqrt{s}<2175 \mathrm{MeV}$, and

$$
\sigma=-3.181 \ln (\sqrt{s})+58.978 \text {, }
$$

for $\sqrt{s}>2175 \mathrm{MeV}$.
[1] W. Gudowski, Nucl. Phys. A654, 436c (1999).

[2] I. S. K. Gardner, In S. Myers et al., editors, EPAC98: Proceedings (IOP Publishing, Bristol, 1998), p. 98.

[3] E. Parker, Space Weather 3, S08004 (2005).

[4] J.-J. Gaimard and K.-H. Schmidt, Nucl. Phys. A531, 709 (1991).

[5] A. R. Junghans, M. de Jong, H. G. Clerc, A. V. Ignatyuk, G. A. Kudyaev, and K.-H. Schmidt, Nucl. Phys. A629, 635 (1998).

[6] A. Boudard, J. Cugnon, S. Leray, and C. Volant, Phys. Rev. C 66, 044615 (2002).

[7] J. Cugnon, T. Mizutani, and J. Vandermeulen, Nucl. Phys. A352, 505 (1981).

[8] Th. Aoust and J. Cugnon, Eur. Phys. J. A 21, 79 (2004).

[9] L. Kisslinger, Phys. Rev. 98, 761 (1955).

[10] M. Krell and T. Ericson, Nucl. Phys. B11, 521 (1969).

[11] G. R. Satchler, Nucl. Phys. A540, 533 (1992).

[12] M. A. E. Akhter, S. A. Sultana, H. M. Sen Gupta, and R. J. Peterson, J. Phys. G 27, 755 (2001).

[13] E. Friedman, Phys. Rev. C 28, 1264 (1983).

[14] S. Jena and S. Swain, Phys. Rev. C 55, 3015 (1997).

[15] M. J. Johnson and G. R. Satchler, Ann. Phys. (NY) 248, 134 (1996).

[16] C. Mahaux and R. Sartor, Adv. Nucl. Phys. 20, 1 (1991).

[17] A. M. Lane, Phys. Rev. Lett. 8, 171 (1962).

[18] T. Ericson and W. Weise, Pions and Nuclei (Clarendon Press, Oxford, 1988).

[19] T.-S. H. Lee and R. P. Redwine, Annu. Rev. Nucl. Part. Sci. 52, 23 (2002).

[20] B. ter Haar and R. Malfliet, Phys. Rev. C 36, 1611 (1987).

[21] J. F. Crawford et al., Phys. Rev. C 22, 1184 (1980).

[22] D. R. F. Cochran et al., Phys. Rev. D 6, 3085 (1972).

[23] N. J. DiGiacomo et al., Phys. Rev. C 31, 292 (1985).

[24] R. D. McKeown et al., Phys. Rev. C 24, 211 (1981).

[25] D. Ashery et al., Phys. Rev. C 23, 2173 (1981).

[26] K. Nakai et al., Phys. Rev. Lett. 44, 1446 (1980).
[27] J. N. Ginocchio, Phys. Rev. C 17, 195 (1978).

[28] J. Martensson et al., Phys. Rev. C 62, 014610 (2000).

[29] Y. Iwamoto et al., Phys. Rev. C 70, 024602 (2004).

[30] H. A. Khan, N. A. Khan, and R. J. Peterson, Phys. Rev. C 35, 645 (1987).

[31] S. Furihata, Nucl. Instrum. Methods B 171, 251 (2000).

[32] S. Furihata and T. Nakamura, J. Nucl. Sci. Technol., Suppl. 2, 720 (2002).

[33] R. J. Peterson, S. de Barros, H. Schechter, S. G. Maschnik, A. G. daSilva, and J. C. Suitta, Eur. Phys. J. A 10, 69 (2001).

[34] A. V. Prokofiev, S. G. Mashnik, and A. J. Sierk, Nucl. Sci. Eng. 131, 78 (1999).

[35] T. Enqvist et al., Nucl. Phys. A686, 481 (2001).

[36] A. K. Kerman, H. McManus, and A. M. Thaler, Ann. Phys. (NY) 8, 551 (1959).

[37] H. Feshbach, A. Gal, and J. Hüfner, Ann. Phys. (NY) 66, 20 (1971).

[38] H. Hirata, F. Lenz, and K. Yazaki, Ann. Phys. (NY) 108, 116 (1977).

[39] H. Hirata, J. H. Koch, F. Lenz, and E. J. Moniz, Ann. Phys. (NY) 120, 205 (1979).

[40] K. Klingenbeck, M. Dillig, and M. G. Huber, Phys. Rev. Lett. 41, 387 (1978).

[41] E. Oset and W. Weise, Nucl. Phys. A329, 365 (1979).

[42] E. Oset and W. Weise, Nucl. Phys. A319, 477 (1979).

[43] E. Oset, L. L. Salcedo, and D. Strottman, Phys. Lett. B165, 13 (1985).

[44] J. Cugnon and P. Henrotte, Eur. Phys. J. A 16, 393 (2003).

[45] S. G. Mashnik, R. J. Peterson, A. J. Sierck, and M. R. Braunstein, Phys. Rev. C 61, 034601 (2000).

[46] K. K. Gudima, S. G. Mashnik, and V. D. Toneev, Nucl. Phys. A401, 329 (1983).

[47] Th. Aoust et al., Nucl. Instrum. Methods A 562, 810 (2006).

[48] Particle Data Group, K. Hagiwara et al., Phys. Rev. D 66, 010001 (2002). 